\title{
Minimal actions of homeomorphism groups
}

\author{
by
}

Yonatan Gutman (Jerusalem)

\begin{abstract}
Let $X$ be a closed manifold of dimension 2 or higher or the Hilbert cube. Following Uspenskij one can consider the action of $\operatorname{Homeo}(X)$ equipped with the compactopen topology on $\Phi \subset 2^{2^{X}}$, the space of maximal chains in $2^{X}$, equipped with the Vietoris topology. We show that if one restricts the action to $M \subset \Phi$, the space of maximal chains of continua, then the action is minimal but not transitive. Thus one shows that the action of $\operatorname{Homeo}(X)$ on $U_{\operatorname{Homeo}(X)}$, the universal minimal space of $\operatorname{Homeo}(X)$, is not transitive (improving a result of Uspenskij). Additionally for $X$ as above with $\operatorname{dim}(X) \geq 3$ we characterize all the minimal subspaces of $V(M)$, the space of closed subsets of $M$, and show that $M$ is the only minimal subspace of $\Phi$. For $\operatorname{dim}(X) \geq 3$, we also show that $(M, \operatorname{Homeo}(X))$ is strongly proximal.
\end{abstract}

1. Introduction. We consider here compact $G$-spaces with $G$ a Polish group and the action assumed to be continuous as a function of both variables. Such a $G$-space $X$ is said to be minimal if $X$ and $\emptyset$ are the only $G$-invariant closed subsets of $X$. By Zorn's lemma each $G$-space contains a minimal $G$-subspace. These minimal objects are in some sense the most basic ones in the category of $G$-spaces. For various topological groups $G$ they have been the object of vast study. Given a topological group $G$ one is naturally interested in describing all of them up to isomorphism. Such a description is given by the following construction: one can show there exists a minimal $G$-space $U_{G}$ unique up to isomorphism such that if $X$ is a minimal $G$-space then $X$ is a factor of $U_{G}$, i.e., there is a continuous $G$-equivariant mapping from $U_{G}$ onto $X$. $U_{G}$ is called the universal minimal $G$-space (for existence and uniqueness see [Usp00]). Calculating this minimal universal space explicitly is very hard. For some groups the space itself is complicated, e.g. by a known theorem the universal minimal flow of a non-compact locally compact group is non-metrizable (see [KPT05, Theorem A2.2]).

2000 Mathematics Subject Classification: Primary 37B05; Secondary 54H15, 22F50. Key words and phrases: homeomorphism group, minimal action, Hilbert cube. This research was supported by the Israel Science Foundation (grant No. 1333/04). 
For non-locally compact groups the universal minimal space may reduce to one point. Such groups are called extremely amenable. Since every $G$-space $X$ contains a minimal $G$-space, these groups may be characterized by a fixed point property, i.e. any $G$-space $X$ has a $G$-fixed point. Using novel and original techniques Pestov [Pes98] showed that the following groups have this property: the group of order preserving automorphisms of the rational numbers, equipped with the topology in which a subbasis of the identity consists of (all) stabilizers of a finite number of points, and the groups $\mathrm{Homeo}_{+}(I)$ and $\mathrm{Homeo}_{+}(\mathbb{R})$ of orientation preserving homeomorphisms of the unit interval and of the real line, respectively, equipped with the compact-open topology. The universal minimal space may be metrizable without being a single point, for example: the circle $S^{1}$ is the universal minimal space of the group $\mathrm{HomeO}_{+}\left(S^{1}\right)$ of its orientation preserving homeomorphisms, equipped with the compact-open topology.

Motivated by the last result Pestov asked in the last section of [Pes98] if the Hilbert cube $Q=[-1,1]^{\mathbb{N}}$ with the natural action of $\operatorname{Homeo}(Q)$, equipped with the compact-open topology, is the universal minimal space for Homeo $(Q)$. In [Usp00] Uspenskij answered Pestov's question in the negative by showing that for every topological group $G$, the action of $G$ on the universal minimal space $U_{G}$ is not 3-transitive, i.e., there exist triples $\left(a_{1}, a_{2}, a_{3}\right)$ and $\left(b_{1}, b_{2}, b_{3}\right)$ of distinct points of $U_{G}$ such that no $g \in G$ satisfies $g\left(a_{i}\right)=b_{i}$ for $i=1,2,3$. To prove this, Uspenskij introduced the space of maximal chains of a given topological space. We now recall this notion.

Given a compact space $K$, let $V(K)$ be the space of all non-empty closed subsets of $K$, equipped with the Vietoris topology (see Definition 1.1 in [IN99]). A subset $C \subset V(K)$ is a chain in $V(K)$ if for any $E, F \in C$ either $E \subset F$ or $F \subset E$. A chain is maximal if it is maximal with respect to the inclusion relation. One verifies easily that a maximal chain in $V(K)$ is a closed subset of $V(K)$, and that $\Phi$, the space of all maximal chains in $V(K)$, is a closed subset of $V(V(K))$, i.e. $\Phi \subset V(V(K))$ is a compact space. Note that a $G$-action on $K$ naturally induces a $G$-action on $V(K)$ and $\Phi(K)$. This is true in particular for $K=U_{G}$. Therefore there is a continuous $G$-equivariant mapping $f: U_{G} \rightarrow \Phi\left(U_{G}\right)$. By cleverly investigating this mapping Uspenskij achieved the aforementioned result.

Motivated by Uspenskij's idea of looking at the maximal chain space of the universal minimal space, Glasner and Weiss [GW03] studied the maximal chain space of the Cantor set $K$, and showed that it is the universal minimal space for $\operatorname{Homeo}(K)$, equipped with the compact-open topology. It is important to point out that whereas Uspenskij used the (abstract) existence of the space of maximal chains in $V\left(U_{G}\right)$, Glasner and Weiss' method is constructive. The first steps are constructing the maximal chain space of the Cantor set and analyzing its properties. 
In a recent article Pestov asked (attributing the questions to Uspenskij) for an explicit description of the universal minimal space of the group $\operatorname{Homeo}(X)$ (equipped with the compact-open topology), $X$ being a closed manifold of dimension 2 or higher or the Hilbert cube (see [Pes05, Section 5.2, Open Questions $28 \& 29]$ ). Here and elsewhere, the term closed manifold refers to a compact manifold without boundary. Motivated by these and similar questions (where $X$ is allowed to be an even more general topological space) we apply the constructive method to a large class of groups of homeomorphisms of topological spaces (equipped with the compact-open topology). This class includes in particular the group of homeomorphisms of any closed manifold of dimension 2 or higher or of the Hilbert cube.

It is important to note that the specific ideas Glasner and Weiss used in [GW03] heavily depend on the fact that $K$ is zero-dimensional. For higher dimensions new ideas are needed. The scheme we would ideally like to use is to start with the given space $X$, then characterize all minimal subspaces of $V(X)$, next characterize the minimal subspaces of $V^{2}(X)=V(V(X))$ and so on. This scheme would include the analysis of the space of maximal chains in $V(X)$ and much more, but unfortunately it turns out to be difficult to carry out.

We managed to obtain results for the "first three levels". It is easy to see that the only minimal subspaces of $V(X)$ are $\{X\}$ and $\{\{x\} \mid x \in X\}$. Characterizing all minimal subspaces of $V^{2}(X)$ already turns out to be hard. However, one encounters a new and interesting phenomenon involving continua, i.e. non-empty compact and connected metric spaces. Indeed, $\Phi$, the space of maximal chains in $V(X)$, is not minimal, but the subspace $M \subset \Phi$ of maximal chains (consisting only) of continua of $X$ is minimal. This space can also be shown to coincide with the space of connected (with respect to $V(X)$ ) maximal chains (see Lemma 2.3). Put formally:

THEOREM 1.1. If $X$ is a closed manifold of dimension 2 or higher, or the Hilbert cube, then $M$, the space of maximal chains of continua, is minimal under the action of $\operatorname{Homeo}(X)$ on $\Phi$.

This theorem enables us to improve on Uspenskij's result by proving:

TheOREM 1.2. If $X$ is a closed manifold of dimension 2 or higher, or the Hilbert cube, and $G=\operatorname{Homeo}(X)$, then the action of $G$ on the universal minimal $G$-space $U_{G}$ is not transitive.

Interestingly, for a large class of spaces $X$ the space $M$ of maximal chains of continua is the only minimal Homeo $(X)$-subspace of $\Phi$. In particular, we prove:

TheOrem 1.3. If $X$ is a closed manifold of dimension 2 or higher, or the Hilbert cube, then $M$, the space of maximal chains of continua, is the only minimal subspace of the $\operatorname{Homeo}(X)$-space $\Phi$. 
Analyzing all minimal subspaces of $V^{3}(X)$ turned out to be difficult. However, we managed to classify all minimal subspaces of $V(M) \subset V^{3}(X)$ :

THEOREM 1.4. If $X$ is a closed manifold of dimension 3 or higher, or the Hilbert cube, then the action of $\operatorname{Homeo}(X)$ on $V(M)$, the space of nonempty closed subsets of the space of maximal chains of continua, has exactly the following minimal subspaces:

(1) $\{M\}$,

(2) $\left\{M_{x}\right\}_{x \in X}$, where $M_{x}=\left\{c \in M(X) \mid \bigcap\left\{c_{\alpha} \mid c_{\alpha} \in c\right\}=\{x\}\right\}$,

(3) $\{\{c\} \mid c \in M\}$.

$M$ is said to be strongly proximal under $G=\operatorname{Homeo}(X)$ if for any Borel probability measure $\mu$ on $M$, there exists a sequence $\left(g_{n}\right) \subset G$ such that $\left[g_{n}\right]_{*}(\mu)$ converges to the measure concentrated at a singleton. We prove:

TheOREM 1.5. If $X$ is a closed manifold of dimension 3 or higher, or the Hilbert cube, then $(M, \operatorname{Homeo}(X))$ is strongly proximal.

Acknowledgements. This paper is part of the author's $\mathrm{PhD}$ thesis under the supervision of Professor Benjamin Weiss. I would like to thank him for his support and advice. I would also like to thank the two referees for a careful reading of the paper and many useful suggestions.

2. Preliminaries. In this paper an effort is made to state theorems and lemmas in their broadest generality. We use the symbol $X$ to denote the space we are working with. $(X, d)$ is always assumed to be compact, metric, connected and non-trivial (by which we mean it contains more than one point, hence it contains infinitely many points). By an $\varepsilon$-net of a set $D \subset X$ we mean a finite collection $A=\left\{a_{i}\right\}_{i=1}^{K} \subset D$ such that for all $p \in D$, $\operatorname{dist}(p, A)=\min _{i=1, \ldots, K} d\left(p, a_{i}\right)<\varepsilon$.

Let $V(X)$ denote the space of non-empty closed subsets of $X$. We equip $V(X)$ with the Hausdorff metric:

$$
\begin{aligned}
d_{V(X)}\left(A_{1}, A_{2}\right) & =d\left(A_{1}, A_{2}\right) \\
& =\inf \left\{\varepsilon>0 \mid A_{2} \subset B\left(A_{1}, \varepsilon\right) \text { and } A_{1} \subset B\left(A_{2}, \varepsilon\right)\right\},
\end{aligned}
$$

where $A_{1}, A_{2}$ are non-empty closed sets and $B(A, \varepsilon)=B_{\varepsilon}(A)=\{x \in X \mid$ $\exists a \in A, d(x, a)<\varepsilon\}$. The Hausdorff metric induces the Vietoris topology on $V(X)$ (see Theorem 3.1 of [IN99]). We define $V^{n}(X)$ for all $n \in \mathbb{N}$ using the natural definition $V^{n}(X)=V\left(V^{n-1}(X)\right)$. A warning is due here: along the paper the notation $d(\cdot, \cdot)$ will be used to denote distances in various metric spaces. The reader should keep in mind that $d(\cdot, \cdot)$ denotes mostly the distance of the underlying space $X$ or the Hausdorff distance in $V(X)$, $V^{2}(X)$ or $V^{3}(X)$, where the choice should be clear from the context. 
Let $C(X)$ be the subspace of $V(X)$ consisting of all subcontinua of $X$. Here are two definitions that will play an important role in the paper:

$$
\begin{gathered}
\Phi(X)=\text { the collection of maximal chains in } V(X), \\
M(X)=\text { the collection of maximal chains in } C(X) .
\end{gathered}
$$

If the underlying space $X$ is clear from the context we write $\Phi, M$ respectively. In Lemma 2.3 we show that $M$ can be characterized as the space of connected (with respect to $V(X)$ ) maximal chains. If $c \in M(X)$ and $D \in c$ then we define the initial segment of $c$ ending at $D$ to be $c^{\prime}=\{R \in c \mid R \subset D\}$. Notice that $c^{\prime} \in M(D)$. Let

$$
r(c)=\bigcap\left\{c_{\alpha} \mid c_{\alpha} \in c\right\}
$$

while the set of all chains rooted at $\{x\}$ is denoted by $M_{x}$ and $\Phi_{x}$, respectively:

$$
M_{x}=\{c \in M \mid r(c)=\{x\}\}, \quad \Phi_{x}=\{c \in \Phi \mid r(c)=\{x\}\} .
$$

Lemma 2.1. For $c=\left\{c_{\alpha}\right\}_{\alpha \in A} \in M$ and $F \subset$ c one has $\mathrm{Cl}_{X}\left(\bigcup_{c_{\alpha} \in F} c_{\alpha}\right) \in c$ and $\bigcap_{c_{\alpha} \in F} c_{\alpha} \in c$.

Proof. This follows from the maximality of $c$ as a subset of $C(X)$.

Given $c=\left\{c_{\alpha}\right\}_{\alpha \in A} \in M$ and $D \in V(X)$ with $r(c) \subset D$ we call the set $c_{D}=\bigcup\left\{c_{\alpha} \mid c_{\alpha} \subseteq D\right\}$ the maximal element of $c$ inside $D$.

LEMMA 2.2. We have the following:

(1) $c_{D} \subseteq D$.

(2) If $N$ is an open set so that $r(c) \subset N$ and $\partial N \neq \emptyset$ then $c_{\bar{N}} \cap \partial N \neq \emptyset$.

Proof. (1) is a direct consequence of Lemma 2.1 and the fact that $D$ is closed.

(2) Let $I=\bigcap\left\{c_{\alpha} \mid c_{\alpha} \cap \partial N \neq \emptyset\right\}$. By standard compactness arguments $I \cap \partial N \neq \emptyset$ and thus it is enough to show $I \subseteq c_{\bar{N}}$. Assume otherwise. Then $c_{\bar{N}} \subsetneq I$. According to Theorem 15.2 of [IN99] there exists $F \in c$ such that $c_{\bar{N}} \subsetneq F \subsetneq I$. Since $F \cap N \supset r(c) \neq \emptyset$ and $F$ is connected, it follows that $F \subset N$ and thus $F \subseteq c_{\bar{N}}$, a contradiction.

Lemma 2.3. $M=\Phi \cap C(V(X))$.

Proof. Let $c \in M$. According to Lemma 14.4 of [IN99], $c$ is an order arc in $C(X)$, i.e., there exists a homeomorphism $i:[0,1] \rightarrow C(X)$ such that $i([0,1])=c$ and $0 \leq t_{1}<t_{2} \leq 1$ implies $i\left(t_{1}\right) \subsetneq i\left(t_{2}\right)$. In particular, $c$ is connected. Hence $M \subset \Phi \cap C(V(X))$. To prove the opposite inclusion assume $c \in \Phi \cap C(V(X))$ and some $D \in c$ is not connected, i.e. $D=D_{1} \cup D_{2}, D_{1}, D_{2}$ disjoint closed sets. Every member of $c$ is either contained in $D_{1}$ or meets $D_{2}$. This implies $c$ is not connected, contradicting the initial assumption. 
Lemma 2.1. $\left\{M_{x}\right\}_{x \in X} \in C(V(M))$ and the function $m: X \rightarrow\left\{M_{x}\right\}_{x \in X}$ given by $m(x)=M_{x}$ is a homeomorphism.

Proof. Recall that $r: M \rightarrow X$ is the continuous function given by $r(c)=$ $\bigcap_{c_{\alpha} \in c} c_{\alpha}$. Notice that for $x \in X, M_{x}=r^{-1}(\{x\})$, which implies $M_{x} \in V(M)$ and the function $m^{-1}: Z \rightarrow X$, where $Z=\left\{M_{x}\right\}_{x \in X} \subset V(M)$, is continuous and 1-to-1. Now, the set $Z$ is closed in $V(M)$ and therefore compact, and thus $m^{-1}$ is a homeomorphism. Hence $m=r^{-1}$ is a homeomorphism, and $X$ being connected, so is $Z$.

3. Local transitivity, strong arcwise-inseparability and strong $\mathbb{R}$ inseparability. In this section we introduce important topological assumptions used throughout the article and discuss some examples. Our actions of a group $G$ on $X$ will always be induced by $G$ being a subgroup of $\operatorname{Homeo}(X)$, equipped with the compact-open topology (which in this setting is the same as the uniform convergence topology, see [Mun75, p. 286]). The action of $G$ on $X$ induces a natural action on $V^{n}(X)$ for all $n \in \mathbb{N}$. Given $g \in G$ and $A \in V(X)$ (i.e. $A \subset X$ is a closed set) one defines $g A=\{g a \mid a \in A\}$. The action of $G$ on $V^{n}$ for general $n$ is defined inductively, based on the equality $V^{n}(X)=V\left(V^{n-1}(X)\right)$. We assume that the group (or the action) is locally transitive in the sense that for any open set $U \subset X$ and $x \in U$ the set $\left\{g x \mid g \in G_{U}\right\}$ is a neighborhood of $x$, where

$$
G_{U}=\{g \in G \mid g x=x \text { for } x \notin U\} .
$$

For a compact interval $I \subset \mathbb{R}$ we denote by $C_{s}(I, X)$ the collection of continuous simple (injective) paths $p: I \rightarrow X$. We call such paths arcs. As is usually done in the literature, the images of arcs are called arcs as well. A space $X$ is called strongly arcwise-inseparable (SAI) if for any nonempty open and connected set $U \subset X$ and any arc $p \in C_{s}([a, b], U)$, the set $U \backslash p([a, b])$ is connected and non-empty. A space $X$ is called strongly $\mathbb{R}$-inseparable (SRI) if for any non-empty open and connected set $U \subset X$ and any arc $p \in C_{s}([a, b], X)$, the set $U \backslash p([a, b])$ is connected and non-empty. Notice that (SRI) implies (SAI). Throughout the article $X$ is assumed to have one of these two properties. Here are the basic facts the reader should keep in mind. Closed manifolds of dimension 2 are strongly arcwise-inseparable (see Theorem A.1). Closed manifolds of dimension 3 or higher and the Hilbert cube are strongly $\mathbb{R}$-inseparable (see Theorem A.3).

We now list some locally transitive groups (except the case of the Hilbert cube, which is proven in Lemma A.2, the other examples are simple and therefore the proofs are omitted):

EXAMPLES 3.1 (Locally transitive groups). Let $X$ be the Hilbert cube or a closed manifold of dimension 2 or higher. Then any group containing one 
of the following groups is locally transitive:

(1) $G=\operatorname{Homeo}_{0}(X)$, the arcwise connected component of the identity in $\operatorname{Homeo}(X)$.

For $X$ an orientable manifold:

(2) $G=\mathrm{Homeo}_{+}(X)$, the group of orientation preserving homeomorphisms.

For $X$ a smooth manifold:

(3) $G=\operatorname{Diffeo}_{0}(X)$, the arcwise connected component of the identity in the group of diffeomorphisms of $X$.

4. The minimal subspaces of $V(X)$. Let $X$ be a Peano continuum (i.e. a metric, compact, connected and locally connected space) with the property that the removal of a point from any open and connected set does not affect its connectedness.

We now characterize the minimal subspaces of $V(X)$. Recall that the action of $G$ on $X$ is called $n$-transitive if $|X| \geq n$ and for any two $n$-tuples of distinct points $\left(a_{1}, \ldots, a_{n}\right),\left(b_{1}, \ldots, b_{n}\right) \in X^{n}$, there exists $g \in G$ such that $g\left(a_{i}\right)=b_{i}$ for $i=1, \ldots, n$.

Lemma 4.1 (Global transitivity). Suppose $X$ is a continuum such that for each connected open set $U \subset X$ and each $p \in X$ the set $U \backslash\{p\}$ is connected. If the action of $G$ on $X$ is locally transitive then for any open and connected set $U \subset X$, the action of $G_{U}$ on $U$ is $n$-transitive for all $n \in \mathbb{N}$.

Proof. By induction. The case $n=1$ follows from the assumption, as given $x \in U$ the set $\left\{g x \mid g \in G_{U}\right\}$ is open and closed in $U$, and hence is equal to $U$. Let now $n>1$. Let $\left(a_{1}, \ldots, a_{n}\right)$ and $\left(b_{1}, \ldots, b_{n}\right)$ be two $n$-tuples of distinct points of $U$. By assumption there exists an $f \in G_{U}$ so that $f\left(a_{i}\right)=b_{i}$ for $i=1, \ldots, n-1$. Let $V=U \backslash f\left(\left\{a_{1}, \ldots, a_{n-1}\right\}\right)$. Then $V$ is open and connected. Apply the case $n=1$ to find a $g \in G_{V}$ so that $g\left(f\left(a_{n}\right)\right)=b_{n}$. Define $h=g \circ f$. Then $h \in G_{U}$ and $h\left(a_{i}\right)=b_{i}$ for $i=1, \ldots, n$.

THEOREM 4.2. Under the assumptions of the preceding lemma the only minimal subspaces of $V(X)$ are:

(1) $\{X\}$,

(2) $\{\{x\} \mid x \in X\}$.

Proof. It is clear that the two given subspaces are minimal. To show they are the only minimal subspaces it is enough to prove that any element of $V(X)$ has the property that the closure of its orbit intersects one of these subspaces. So let $A \in V(X)$. If $|A|<\infty$ then by Lemma 4.1 one can find $g_{n} \in G, n \in \mathbb{N}$, and $z \in X$ so that $g_{n}(A) \rightarrow\{z\}$ as $n \rightarrow \infty$, i.e. the closure of the orbit of $A$ intersects the second subspace. If $|A|=\infty$ we will show 
that the closure of the orbit of $A$ intersects the first subspace. Let $\varepsilon>0$ and let $\left\{x_{i}\right\}_{i=1}^{l} \subset X$ be an $\varepsilon$-net of $X$. Find $\left\{y_{i}\right\}_{i=1}^{l} \subset A$ and $g \in G$ such that $g\left(y_{i}\right)=x_{i}$. Then $d(g(A), X)<\varepsilon$.

5. Approximation of $M$ by ray-induced chains. From now on we assume that $X$ is a strongly arcwise-inseparable Peano continuum. In this section we will show that chains in $M$ can be approximated by so-called ray-induced chains.

Definition 5.1. We call the members of $C_{s}([0, \infty), X)$ rays. By an $\overline{\mathbb{R}}_{+-}$ chain we mean any element $c$ of $M$ such that $c=\left(c_{t}\right)_{t \in[0, \infty]}$ and there exists a ray $\gamma$ with $c_{t}=\gamma([0, t])$ for all $t<\infty$. When the last condition is satisfied, we say that $c$ is induced by $\gamma$. Observe that then $c_{\infty}=X$ (this follows from the maximality of $c$ ) and thus $\gamma$ is dense in $X$ (in the sense that $\gamma([0, \infty)$ ) is dense in $X$ ), again by maximality. We define

$$
\mathfrak{R}=\left\{c \in M \mid c \text { is an } \overline{\mathbb{R}}_{+} \text {-chain }\right\} \quad \text { and } \quad \mathfrak{R}_{x}=\mathfrak{R} \cap M_{x} \quad(x \in X) .
$$

Lemma 5.2. Let $\gamma \in C_{s}([0, k], U)$, where $U$ is an open connected set in $X$. Then given $\varepsilon>0$ and $x_{1}, \ldots, x_{l} \in U$ there exists an arc $\gamma^{\prime} \in C_{s}([0, k+l], U)$ such that $\gamma^{\prime}(t)=\gamma(t)$ for $t \leq k-1, d\left(\gamma^{\prime}(t), \gamma(t)\right)<\varepsilon$ for $t \in[k-1, k]$ and $x_{1}, \ldots, x_{l} \in B_{\varepsilon}\left(\gamma^{\prime}([k, k+l])\right)$.

Proof. An easy induction shows that it suffices to consider the case when $l=1$. If $x_{1} \in \gamma([0, k])$ we are done, so assume otherwise. Pick $s \in(k-1, k)$ with diam $\gamma([s, k])<\varepsilon / 2$, and using the connectedness of the set $U \backslash \gamma([0, s])$ pick an arc $p$ in it from $\gamma(k)$ to $x_{1}$. The desired arc $\gamma^{\prime}$ is obtained by first traveling along $\gamma$ until we hit $p$, and then along $p$. (Parametrization needs to be adjusted so that $\gamma^{\prime}(t)=\gamma(t)$ for $t \leq s$ and $\gamma(t)$ stays close to $\gamma(s)=\gamma^{\prime}(s)$ for $t \in[s, k])$.

Theorem 5.3. $\overline{\mathfrak{R}}=M$ (for $X$ a Peano continuum which is $S A I)$.

Proof. Let $c \in M$ and $\varepsilon>0$. By Lemma 14.4 of [IN99] there is an embedding $j$ of $[0, \infty]$ into $C(X)$ such that $c=j([0, \infty])$ and $j\left(t_{1}\right) \subset j\left(t_{2}\right)$ for $t_{1}<t_{2}$. Subdivide $[0, \infty]$ into infinitely many intervals, each mapped under $j$ to a set of diameter $<\varepsilon$. By changing the units in the domain one can assume that the intervals have diameter less than 1 and thus $d(j(t), j(k))<\varepsilon$ for all reals $t \geq 0$ and integers $k$ such that $k-1 \leq t \leq k$. Denote by $\operatorname{Con}_{\varepsilon}(j(k)) \subset$ $B_{\varepsilon}(j(k))$ the connected component of $B_{\varepsilon}(j(k))$ which contains $j(k)$; it is open in $X$ by the local connectedness of $X$. Inductively we construct arcs $\gamma_{k}:[0, k] \rightarrow X$ such that:

(1) $\left\{\gamma_{k}(0)\right\}=r(c), \gamma_{k}([k-1, k]) \subset \operatorname{Con}_{\varepsilon}(j(k))$ and $j(k) \subset B_{\varepsilon / k}\left(\gamma_{k}([k-1, k])\right)$, 
(2) $\gamma_{k}(t)=\gamma_{k-1}(t)$ for $t \in[0, k-2]$,

(3) $d\left(\gamma_{k}(t), \gamma_{k-1}(t)\right)<\varepsilon$ for $t \in[k-2, k-1]$.

To this end suppose first that $k \geq 2$ and $\gamma_{k}$ has already been constructed. Let $U=\operatorname{Con}_{\varepsilon}(j(k))$. Let $x_{1}, \ldots, x_{l}$ be an $\varepsilon / k$-net of $j(k)$. Applying Lemma 5.2 with $\gamma=\gamma_{k}$ and then changing the parameter set of $\gamma^{\prime}$ from $[0, k+l]$ to $[0, k+1]$ we get the desired arc $\gamma_{k+1}$. This takes care of the inductive step; the case $k=1$ is handled similarly. (Conditions (2) and (3) are then void.) Put $\gamma(t)=\lim _{k \rightarrow \infty} \gamma_{k}(t)$ and $a_{t}=\gamma([0, t])$ for $t \geq 0$. Since $\bigcup_{k} j(k)=X$ we infer from (1) that the image of $\gamma$ is dense in $X$. Moreover, $a_{k}=\gamma_{k+1}([0, k])$ by (2), and thus from (1), (3) and the monotonicity of the sequence $(j(n))$ it follows that $a_{k}$ is contained in the $2 \varepsilon$-neighborhood of $j(k)$ and contains the set $S=\gamma_{k+1}([k-1, k])$ such that $j(k) \subset B_{2 \varepsilon}(S)$. Hence $d\left(a_{k}, j(k)\right)<2 \varepsilon$ for all $k$. Now if $t \geq 0$, say $t \in[k, k+1]$ for some integer $k$, then

$$
\begin{gathered}
a_{t} \subset a_{k+1} \subset B_{2 \varepsilon}(j(k+1)) \subset B_{3 \varepsilon}(j(t)), \\
\left.B_{3 \varepsilon}\left(a_{t}\right)\right) \supset B_{3 \varepsilon}\left(a_{k}\right) \supset B_{\varepsilon}(j(k)) \supset j(t) .
\end{gathered}
$$

Thus the ray-induced chain $\left\{a_{t}\right\}_{t \geq 0} \cup\{X\}$ is $3 \varepsilon$-close to $c$, completing the proof.

Until now we have assumed that $X$ is strongly arcwise-inseparable. Suppose now $X$ is strongly $\mathbb{R}$-inseparable. Under this assumption we first present a lemma which is a generalization of Lemma 5.2, and then a definition which is a generalization of Definition 5.1. Finally, we state Theorem 5.6 which generalizes Theorem 5.3. The proof of Theorem 5.6 is omitted as it can be supplied by a reader who has understood the proof of Theorem 5.3.

Lemma 5.4. Let $\gamma_{1}, \ldots, \gamma_{N} \in C_{s}\left([0, k], U_{i}\right)$ be disjoint arcs, where $U_{1}, \ldots, U_{N}$ are open connected sets in $X$. Then, given $\varepsilon, \delta>0$, there exist $a>k$ and disjoint arcs $\gamma_{i}^{\prime} \in C_{s}\left([0, a], U_{i}\right)$ such that $\gamma_{i}^{\prime}(t)=\gamma_{i}(t)$ for $t \leq k-\delta, d\left(\gamma_{i}^{\prime}(t), \gamma_{i}(t)\right)<2 \varepsilon$ for $t \in[k-\delta, k]$ and each arc $\gamma_{i}^{\prime}([0, a])$ is $\varepsilon / 2$-dense in $X$.

Moreover, if for all $i$ one has $\operatorname{diam} U_{i}<\varepsilon$ and $\gamma_{i}(k) \in U_{i} \backslash F_{i}$ for some closed set $F_{i}$ with $U_{i} \backslash F_{i}$ connected, then the arcs $\gamma_{i}^{\prime}$ can be constructed so that $\gamma_{i}^{\prime}(t) \notin F_{i}$ for $t \geq k-\delta$.

Proof (sketch). By considering the arcs $t \mapsto \gamma_{i}(C t)$ for $C$ large enough, and eventually switching back to the original parametrization, one can assume that $\delta=1$. The first part is then proved as in Lemma 5.2, using the (SRI) property of $X$ to make the approximating arcs disjoint and taking for $x_{1}, \ldots, x_{l}$ an $\varepsilon / 2$-net in $X$.

The idea of the proof of the "moreover" part is first to use the above with $\varepsilon$ replaced by $\varepsilon / 2$ and then, for each $i$, to subdivide the segment $[k-\delta, a]$ into finitely many segments, so small that they are mapped by $\gamma_{i}^{\prime}$ into $X \backslash F_{i}$ 
or into $U_{i}$. Then, possibly combining adjacent segments which are mapped to $U_{i}$, one can assume that their endpoints are mapped to $U_{i} \backslash F_{i}$. The connectedness of $U_{i} \backslash F_{i}$ now allows us to modify $\gamma_{i}^{\prime}$ on such a segment so that the altered $\gamma_{i}^{\prime}$ takes values in $U_{i} \backslash F_{i}$. Since diam $U_{i}<\varepsilon$, the modifications will stay $\varepsilon$-close to the unaltered $\gamma_{i}^{\prime}$ 's, and hence $2 \varepsilon$-close to $\gamma_{i}$ on $[k-\delta, k]$.

Definition 5.5. Let $N \in \mathbb{N}$. Equip $M^{N}$ with the product topology. We define the subspace $\mathfrak{R}_{*}^{N} \subset M^{N}$ as follows: $\left(c_{1}, \ldots, c_{N}\right) \in \mathfrak{R}_{*}^{N}$ iff:

(1) $c_{i}=\left\{\gamma_{i}([0, t])\right\}_{t \in \mathbb{R}_{+}} \cup\{X\} \in \mathfrak{R}, i=1, \ldots, n$,

(2) $\gamma_{i}\left(\mathbb{R}_{+}\right) \cap \gamma_{j}\left(\mathbb{R}_{+}\right)=\emptyset, 1 \leq i<j \leq n$.

Theorem 5.6. $\overline{\mathfrak{R}_{*}^{N}}=M^{N}$ (for $X$ a Peano continuum which is $\left.S R I\right)$.

6. The minimality of $M$. Let $X$ be a Peano continuum which is strongly arcwise-inseparable. Our goal in this section is to show that $M$ is minimal under the action of $G$. We start with a definition:

Definition 6.1. $T$ is a $\delta$-tube for $p \in C_{s}([0,1], X)$ if for some $0=t_{0}<$ $t_{1}<\cdots<t_{l}=1$ there exist open connected subsets $U_{1}, \ldots, U_{l}$ (the "links" of the tube) such that $T=\bigcup_{i=1}^{l} U_{i}$ and

(1) $\operatorname{diam} U_{i}<\delta$ and $p\left(\left[t_{i-1}, t_{i}\right]\right) \subset U_{i}$ for $i=1, \ldots, l$,

(2) $\mathrm{Cl}_{X}\left(U_{i}\right) \cap \mathrm{Cl}_{X}\left(U_{j}\right) \neq \emptyset$ iff $|i-j| \leq 1$.

Lemma 6.2. Let $p \in C_{s}([0,1], X)$ and $\delta>0$. Then $p$ has a $\delta$-tube contained in a given neighborhood $U$ of $p([0,1])$.

Proof. Using simplicity of $p$ we may choose $0=t_{0}<\cdots<t_{l}=1$ such that $\operatorname{diam} p\left(\left[t_{i-1}, t_{i}\right]\right)<\delta / 2$ for each $i=1, \ldots, k$. Then there is a $\varrho<\delta / 2$ such that for $B_{i}=B\left(p\left(\left[t_{i-1}, t_{i}\right]\right), \varrho\right)$ we have $B_{i} \cap B_{j}=\emptyset$ for $|i-j|>1$. We define $U_{i}$ to be the connected component of $p\left(\left[t_{i-1}, t_{i}\right]\right)$ in $B_{i}$. By local connectedness, each $U_{i}$ is open and so $T=\bigcup_{i} U_{i}$ is a $\delta$-tube for $p$. Also, if $\delta$ is small enough then $T \subset U$.

Introduce the notation:

$$
\operatorname{dist}(A, B)=\inf _{x \in A, y \in B} d(x, y) \quad \text { and } \quad \operatorname{dist}(x, A)=\operatorname{dist}(\{x\}, A)
$$

for $A, B \subset X$. Notice that in general $\operatorname{dist}(x, A)<d(\{x\}, A)$ and $\operatorname{dist}(A, B)<$ $d(A, B)$.

Lemma 6.3. Let $T=\bigcup_{i=1}^{n} U_{i}$ be an $\varepsilon$-tube around an arc $p:[0, a] \rightarrow X$. Then:

(1) For every continuum $K \subset T$ such that $p(0) \in K$ there exists a $t \in$ $[0, a]$ with $d(K, p([0, t]))<\varepsilon$. 
(2) If $q:[0, b] \rightarrow T$ is an arc satisfying $q(0)=p(0)$ and $q(b)$ belongs to a sufficiently small neighborhood of $p(a)$, then for each $s \in[0, a]$ there exists a $t \in[0, b]$ with $d(p([0, s]), q([0, t]))<\varepsilon$.

(3) If a chain $c \in M_{p(0)}$ is such that $p(a)$ belongs to a sufficiently small neighborhood of $C \subset T$ for some $C \in c$, then given $s \in[0, a]$ there exists a $C^{\prime} \in c$ such that $d\left(p([0, s]), C^{\prime}\right)<2 \varepsilon$.

Proof. (1) Let $j=n$ if $K \cap U_{n} \neq \emptyset$ and $j=\min \left\{i \mid K \cap U_{i}=\emptyset\right\}$ otherwise. Then $t=t_{j}$ does the job.

(2) For the above mentioned sufficiently small neighborhood of $p(a)$ we take $U_{n}$ from the definition of a tube. Let first $s=t_{j}$ for some $j \geq 1$. Then, with $t=\inf \left\{t^{\prime} \in[0, b] \mid q\left(t^{\prime}\right) \notin U_{j-1}\right\}$, it is easy to see that $d(q([0, t])$, $\left.p\left(\left[0, t_{j}\right]\right)\right)<\varepsilon$. The general case follows similarly.

(3) Let $B_{2 \delta}(p(a))$ be a sufficiently small neighborhood of $p(a)$ as desired in (2) for some $0<\delta<\varepsilon$. By Theorem 5.3 there exists a chain $c^{\prime} \in M_{p(0)}$ induced by a ray $\gamma:[0,1] \rightarrow X$ with dense image and $\gamma(0)=p(0)$, such that $d\left(c, c^{\prime}\right)<\delta$; here we require that $\delta<\operatorname{dist}(p([0, a]), X \backslash T)$. Then for some $q>0$ one has $\gamma([0, q]) \subset T$ and $d(p(a), \gamma(q))<2 \delta$. By (2), there exists a $t \in[0, q]$ such that $d(p([0, s]), \gamma([0, t]))<\varepsilon$. Thus it remains to choose $C^{\prime}$ so that $d\left(\gamma([0, q]), C^{\prime}\right)<\varepsilon$.

Recall $M_{x}=\{c \in M \mid r(c)=\{x\}\}$.

Lemma 6.4. Let $x \in X, \varepsilon>0$ and $c \in M_{x}$. Set $f=\{\gamma([0, t])\}_{t \in \mathbb{R}_{+}} \cup$ $\{X\} \in \Re_{x}$. Let $a \in \mathbb{R}$ be such that $d(X, \gamma([0, a]))<\varepsilon$. Let $U \subset X$ be an open subset such that $\gamma([0, a]) \subset U$. Then there is $g \in G_{U}$ with $d(g(c), f)<3 \varepsilon$.

Proof. As $\gamma_{\mid[0, a]} \subset U$ is a simple curve, Lemma 6.2 tells us that $\gamma_{\mid[0, a]}$ has an $\varepsilon$-tube $T$ in $U$. Using Lemma 2.2 one can choose $C \in c$ so that $C \neq\{x\}$ and $C \subset T$. Choose $q \in C \backslash r(c)$. Using Lemma 4.1 choose $g \in G$ so that $g(q)=\gamma(a), g(\gamma(0))=\gamma(0)$ and $g_{\mid T^{c}}=\mathrm{Id}$, which implies $g_{\mid U^{c}}=\mathrm{Id}$. In an imprecise manner one can say that $g$ "stretches" $C$ along $\gamma([0, a])$. Therefore it should not come as a surprise that we will now be able to show that $d(g(c), f)<3 \varepsilon$. In fact, this inequality follows directly from parts (1) and (3) of Lemma 6.3 if one takes into account that for $s>a$ and $D \in c$ with $C \subset D$ the sets $\gamma([0, s])$ and $g(D)$ are $2 \varepsilon$-dense in $X$ (the latter contains $g(C)$ which contains a set $3 \varepsilon$-close to $\gamma([0, a]))$.

TheOREm 6.5. Let $G$ act locally transitively on a Peano continuum $X$ which is strongly arcwise-inseparable. Then the action of $G$ on $M(X)$ is minimal.

Proof. Let $c, f \in M$ and $\varepsilon>0$. Using transitivity of $G$ (Lemma 4.1) one can assume that $\{r\}=r(c)=r(f)$. By Theorem 5.3 one can assume $f=\{\gamma([0, t])\}_{t \in \mathbb{R}_{+}} \cup\{X\} \in \mathfrak{R}$. Choose $a>0$ so that $d(\gamma([0, a]), X)<\varepsilon$. 
Now invoke Lemma 6.4 with $U=X$ to conclude there is a $g \in G$ such that $d(g(c), f)<3 \varepsilon$.

COROLlary 6.6. Under the assumptions of Theorem 6.5, the action of $G$ on the universal minimal $G$-space $U_{G}$ is not 1-transitive.

Proof. It is enough to show that the minimal $G$-space $M$ is not 1transitive. Let $c \in \mathfrak{R}$; it is induced by some ray $\gamma$. Let $r \in X$. Define $v=\{\bar{B}(r, t)\}_{t \in \mathbb{R}}$. Since no arc is SAI it is easy to show that one cannot map balls $\bar{B}(r, t)$ (homeomorphically) onto arcs of the form $\gamma([0, a])$. This implies there is no $g \in G$ such that $g(v)=c$, so the action of $G$ on $M$ is not 1-transitive.

\section{The uniqueness of $M$ as a minimal subspace of $\Phi$}

Definition 7.1. Let $x \in X, \varepsilon>0$, and $N \in \mathbb{N}$. A sequence $\mathfrak{B}$ of open sets $x \in B_{1} \subset \bar{B}_{1} \subset B_{2} \subset \cdots \subset \bar{B}_{N-1} \subset B_{N}$ is an $(N, \varepsilon)$ annuli telescope around $x$ if there exists an open set $U$ with $\bar{B}_{N} \subset U \subset B(x, \varepsilon)$ such that $U \backslash \bar{B}_{i}$ is connected for $i=1, \ldots, N$. Notice that if $V$ is open and connected with $U \subset V$ then $V \backslash \bar{B}_{i}$ is connected for $i=1, \ldots, N$. For convenience one defines $A_{1}=\bar{B}_{1}$ and $A_{i}=\bar{B}_{i} \backslash \bar{B}_{i-1}, i=2, \ldots, N$. Then $\left\{A_{i}\right\}_{i=1}^{N}$ is called the accompanying telescope decomposition.

Moreover, we say that $c \in \Phi_{x}$ is $\mathfrak{B}$-compatible if there exist $\left\{C_{i}\right\}_{i=1}^{N} \subset c$ such that $\{x\}=C_{1} \subset \cdots \subset C_{N}, C_{i} \subset \bar{B}_{i}$ and $C_{i} \cap A_{i} \neq \emptyset$ for $i=1, \ldots, N$.

We say that $X$ has the telescoping annuli property if for any $x \in X$ and $\varepsilon>0$ there is a $(1, \varepsilon)$ annuli telescope around $x$, which implies by a simple argument that for any $\varepsilon>0$ and $N \in \mathbb{N}$ there is an $(N, \varepsilon)$ annuli telescope around $x$.

Theorem 7.2. If $X$ is a Peano continuum which is $S A I$ and has the telescoping annuli property, then the only minimal subspace of $\Phi(X)$ is $M(X)$.

Proof. Let $c \in \Phi$. Our goal will be to show that the closure of the orbit of $c$ intersects $M$. Let $\varepsilon>0$. Let $f=\{\gamma([0, t])\}_{t \in \mathbb{R}_{+}} \cup\{X\} \in \mathfrak{R}_{r(c)}$. Let $a \in \mathbb{R}$ with $d(X, \gamma([0, a]))<\varepsilon$. Let $T=\bigcup_{i=1}^{N} U_{i}$ be an $\varepsilon$-tube of $\gamma([0, a])$ with $\operatorname{diam} U_{i}<\varepsilon$ and $0=t_{0}<t_{1}<\cdots<t_{l}=a$ such that $p\left(\left[t_{i-1}, t_{i}\right]\right) \subset U_{i}$. Define $T_{k}=\bigcup_{i=1}^{k} U_{i}, k=1, \ldots, N$. As $X$ has the telescoping annuli property one can choose $\mathfrak{B}_{N}=\left\{B_{i}\right\}_{i=1}^{N}$ to be an annuli telescope around $r(c)$ such that $r(c) \subset B_{1} \subset \cdots \subset B_{N} \subset V \subset U_{1}$, with $V$ open and $V \backslash \bar{B}_{i}$ connected for $i=1, \ldots, N$. Let $\left\{A_{i}\right\}_{i=1}^{N}$ be its accompanying telescope decomposition.

By induction we will find $g_{N} \in G$ such that $g_{N}(c)$ is $\mathfrak{B}_{N}$-compatible. Define $\mathfrak{B}_{k}=\left\{B_{i}\right\}_{i=1}^{k}$ for $k=1, \ldots, N$. Notice that for $g_{1}=\mathrm{Id}, g_{1}(c)$ is $\mathfrak{B}_{1^{-}}$ compatible. This is the base step of the induction. Assume we have found $g_{k} \in G, k<N$, such that $g_{k}(c)$ is $\mathfrak{B}_{k}$-compatible. We will now construct 
$g_{k+1} \in G$ so that $g_{k+1}(c)$ is $\mathfrak{B}_{k+1}$-compatible. Let $g_{k}\left(C_{1}\right) \subset g_{k}\left(C_{2}\right) \subset \cdots \subset$ $g_{k}\left(C_{k}\right) \in g_{k}(c)$ with $g_{k}\left(C_{i}\right) \subset \bar{B}_{i}$ and $g_{k}\left(C_{i}\right) \cap A_{i} \neq \emptyset$ for $i=1, \ldots, k$. Let $R=$ $\left[g_{k}(c)\right]_{\bar{B}_{k+1}}$. If $R \cap A_{k+1} \neq \emptyset$, let $g_{k+1}=g_{k}$ and $C_{k+1}=g_{k+1}^{-1}(R)$. If $R \cap A_{k+1}$ $=\emptyset$, define $R_{+}=\bigcap\left\{g_{k}\left(c_{\alpha}\right) \mid g_{k}\left(c_{\alpha}\right) \cap B_{k+1}^{c} \neq \emptyset\right\}$. As $\operatorname{dist}\left(B_{k+1}^{c}, \bar{B}_{k}\right)>0$, the maximality of $c$ implies that $R_{+}=R \cup\{p\}$ for some $p \in B_{k+1}^{c}$. By Lemma 4.1, as $X \backslash \bar{B}_{k}$ is connected one can find $h \in G$ and $y \in A_{k+1}$ such that $h(p)=y$ and $h_{\mid \bar{B}_{k}}=$ Id. Define $g_{k+1}=h \circ g_{k}$. Notice that $g_{k+1}\left(C_{i}\right)=g_{k}\left(C_{i}\right)$ for $i=1, \ldots, k$. Moreover, $g_{k+1} \circ g_{k}^{-1}\left(R_{+}\right) \subset \bar{B}_{k+1}$ and $g_{k+1}\left(R_{+}\right) \cap A_{k+1} \neq \emptyset$. This finishes the induction.

We now choose distinct $y_{i} \in g_{N}\left(C_{i}\right) \cap A_{i}$ and $z_{i} \in U_{i}, i=1, \ldots, N$. Set $B_{0}=\emptyset$. As $T_{k} \backslash \bar{B}_{k-1}$ is open and connected, using property (SAI) one can choose disjoint $\operatorname{arcs} p_{k} \in C_{s}\left([0,1], T_{k} \backslash \bar{B}_{k-1}\right)$ with $p_{i}(0)=y_{i}$ and $p_{i}(1)=z_{i}$. Then one can find disjoint open connected subsets $p_{i}([0,1]) \subset$ $W_{i} \subset T_{k} \backslash \bar{B}_{k-1}, i=1, \ldots, N$, and therefore by Lemma 4.1 we can find $q_{i} \in G$ so that $q_{i}\left(y_{i}\right)=z_{i}$ and $\left[q_{i}\right]_{\mid W_{i}^{c}}=\mathrm{Id}$. Let $q=q_{1} \circ \cdots \circ q_{N} \circ g_{N}$. Notice that $q\left(C_{i}\right) \subset T_{i} \subset B\left(\gamma\left(\left[0, t_{i}\right]\right), \varepsilon\right) \subset B\left(q\left(C_{i}\right), 2 \varepsilon\right)$ for $i=1, \ldots, N$. We claim this implies $d(q(c), f)<2 \varepsilon$. Indeed, for $D \in c$ with $C_{i} \subset D \subset C_{i+1}$, $d\left(q(D), \gamma\left(\left[0, t_{i}\right]\right)\right)<2 \varepsilon$. For $t_{i} \leq s \leq t_{i+1}$ one has $d\left(\gamma([0, s]), q\left(C_{i}\right)\right)<2 \varepsilon$. For $s \geq a$ one has $d(\gamma([0, s]), X)<\varepsilon$. Finally, for $C_{N} \subset D$ for $D \in c$ one has $d(\gamma([0, a]), D)<2 \varepsilon$.

8. The minimal subspaces of $V(M)$. In this section we assume $X$ is a strongly $\mathbb{R}$-inseparable Peano continuum. For Lemma 8.4, Corollary 8.5 and Theorem 8.6 we assume $(X, G)$ has the boundary shrinking property (to be defined in this section). Our goal is to find all minimal subspaces of $V(M)$. Three minimal subspaces are evident: $S_{s}=\{M\}, S_{f}=\left\{M_{x}\right\}_{x \in X}$ and $S_{p}=\{\{c\} \mid c \in M\}$. The surprising conclusion of this section is that these are the only minimal subspaces of $V(M)$. For $F \in V(M)$ let

$$
G F=\{g(F) \mid g \in G\}, \quad r(F)=\{r(c) \mid c \in F\} .
$$

In order to facilitate the statement of various theorems we call $F \in V(M)$ space-like, fiber-like or point-like if respectively $\mathrm{Cl}_{V(M)}(G F) \cap S_{s} \neq \emptyset$, $\mathrm{Cl}_{V(M)}(G F) \cap S_{f} \neq \emptyset$ or $\mathrm{Cl}_{V(M)}(G F) \cap S_{p} \neq \emptyset$. We start with an easy lemma:

Lemma 8.1. If $|r(F)|=\infty$ then $F$ is space-like.

Proof. Let $F \subset V(M)$ be such that $|r(F)|=\infty$. Let $\varepsilon>0$. Let $\left\{f^{i}\right\}_{i=1}^{N} \subset$ $M$ be an $\varepsilon$-net of $M$. Using Lemma 2.1, as $X$ is non-trivial and connected, one can assume that $r\left(f^{1}\right), \ldots, r\left(f^{N}\right)$ are distinct. Choose $c^{i} \in F, i=1, \ldots, N$, such that $r\left(c^{i}\right)=\left\{r^{i}\right\}, i=1, \ldots, N$, are distinct. Using the $N$-transitivity of $G$ (Lemma 4.1) one can assume that $r\left(f^{i}\right)=\left\{r^{i}\right\}$. 
We will now find $g \in G$ so that $d\left(g\left(c^{i}\right), f^{i}\right)<3 \varepsilon$ for $i=1, \ldots, N$. By Lemma 5.6 one can assume $\left(f^{1}, \ldots, f^{N}\right) \in \mathfrak{R}_{*}^{N}$, in particular $f^{i}=$ $\left\{\gamma^{i}([0, t])\right\}_{t \in \mathbb{R}_{+}} \cup\{X\} \in \mathfrak{R}, i=1, \ldots, N, \gamma^{i} \in C_{s}\left(\mathbb{R}_{+}, X\right)$. Let $a \in \mathbb{R}_{+}$ with $d\left(\gamma^{i}([0, a]), X\right)<\varepsilon, i=1, \ldots, N$. Find disjoint open sets $U_{1}, \ldots, U_{N}$ such that $\gamma^{i}([0, a]) \subset U^{i}$. Lemma 6.4 yields $g^{j} \in G_{U_{j}}, j=1, \ldots, N$, such that $d\left(g^{j}\left(c^{j}\right), f^{j}\right)<3 \varepsilon$. Define $g=g^{1} \circ \cdots \circ g^{N}$. Then $d\left(g\left(c^{j}\right), f^{j}\right)<3 \varepsilon, j=$ $1, \ldots, N$. Hence $d_{V(M)}(G(F), M)<4 \varepsilon$.

Definition 8.2. Let $\varepsilon>0$. A non-empty connected open set $A$ is said to $\varepsilon$-encircle a connected closed subset $B \subset X$ if $A \cap B=\emptyset$ and $A \cup B$ is open and connected with $\operatorname{diam}(B \cup A)<\varepsilon$. Notice that the fact that $A \cup B$ is open implies $\partial B \subset \partial A$ and thus indeed " $A$ encircles $B$ ".

Definition 8.3. Let $\varepsilon>0$ and $x \in X$. An open connected subset $A \subset X$ has the $(G, \varepsilon, x)$-boundary shrinking property if:

- The boundary $\partial A$ is connected and has at least two points.

- For any closed $W \subsetneq \partial A, \delta>0$ and $y \in A$ with $y \neq x$, there exists $h \in G_{B(A, \delta)}$ such that $h(x)=x$ and $h(W) \subset B(y, \delta)$.

- There exists an open connected set $E$ which $\varepsilon$-encircles $\bar{A}$.

We say that the $G$-space $X$ has the boundary shrinking property (BSP) if for any $x \in X$ and $\varepsilon>0$ there exists an open connected set $A$ with $x \in A$ which has the $(G, \varepsilon, x)$-boundary shrinking property.

Lemma 8.4. Let the Peano continuum $X$ be SRI and let $(X, G)$ have the boundary shrinking property. Suppose $x \in X$ and $F \in V\left(M_{x}\right)$. Then $F$ is either point-like or fiber-like.

Proof. Let $\varepsilon>0$ and $e \in M$. We say $F$ is $(\varepsilon, e)$-point-like if there exists $g \in G$ such that $d(g(F),\{e\})<\varepsilon$, and $\varepsilon$-fiber-like if there exists $g \in G$ such that $d\left(g(F), M_{x}\right)<\varepsilon$ for some $x \in X$. We will prove that for a given $\varepsilon>0$, $F$ is either $3 \varepsilon$-fiber-like or $(2 \varepsilon, e(\varepsilon))$-point-like for some $e(\varepsilon) \in M$. This will of course imply the statement of the lemma. Let $\varepsilon>0$. Using property (BSP) of $(X, G)$ choose $B$ with the $(G, \varepsilon, x)$-boundary shrinking property. Let $V$ be an open connected set $\varepsilon$-encircling $\bar{B}$. Choose $Z$ with the $(G, \varepsilon / 2, x)$-boundary shrinking property. Let $A$ be an open connected set $\varepsilon / 2$-encircling $\bar{Z}$. We arrange so that $\mathrm{Cl}_{X}(Z \cup A) \subset B$, which implies there is $\delta_{1}>0$ such that $B\left(Z, \delta_{1}\right) \subset B$. Let $f=\left\{f_{\kappa}\right\}_{\kappa \in K} \in F$. Recall $f_{\bar{Z}}=\bigcup\left\{f_{\alpha} \mid f_{\alpha} \subset \bar{Z}\right\}$. Define $S(f)=f_{\bar{Z}} \cap \partial Z$. By Lemma 2.2, $S(f) \neq \emptyset$. Define $H=\{f \in F \mid S(f) \neq \partial Z\}$. We first assume $H \neq \emptyset$. Let $f_{1}, \ldots, f_{N} \in F$. Define

$$
\begin{gathered}
I\left(f_{1}, \ldots, f_{N}\right)=\left\{f \in H \mid \exists i \in\{1, \ldots, N\} S\left(f_{i}\right) \cap S(f) \neq \emptyset\right\}, \\
I^{c}\left(f_{1}, \ldots, f_{N}\right)=H \backslash I\left(f_{1}, \ldots, f_{N}\right) .
\end{gathered}
$$

We are now going to choose a sequence of distinct elements $\left\{f_{i}\right\}_{i=1}^{N} \subset F$ where $N \in \mathbb{N} \cup\{\infty\}$. Start by choosing an arbitrary $f_{1} \in H$. If $I^{c}\left(f_{1}\right)=\emptyset$ 
stop. If not choose $f_{2} \in I^{c}\left(f_{1}\right)$. Clearly $f_{2} \neq f_{1}$. If $I^{c}\left(f_{1}, f_{2}\right)=\emptyset$ stop. If not choose $f_{3} \in I^{c}\left(f_{1}, f_{2}\right)$. Continue in this manner. The inductive process results in one of the following two possibilities: (1) $N \in \mathbb{N}$, (2) $N=\infty$.

In case (1) we claim that $F$ is $(2 \varepsilon, e(\varepsilon))$-point-like for some $e(\varepsilon) \in M$. Let $W=\bigcup_{i=1}^{N} S\left(f_{i}\right)$ be a closed set. Notice that as $\partial Z$ is connected and $S\left(f_{i}\right) \cap S\left(f_{j}\right)=\emptyset$ for $1 \leq i<j \leq N$, we have $W \subsetneq \partial Z$. As $\bar{B} \cup V$ is open and connected, one can find $\zeta \in C_{s}([0,1], \bar{B} \cup V)$ with $\zeta(0)=x$ and $\zeta(1) \in V$ (in particular $\zeta(1) \notin \bar{B}$ ). Invoke Lemma 5.4 (with $k=1$, $F_{1}=\bar{B}$ and $\left.U_{1}=\bar{B} \cup V\right)$ to find $\bar{\zeta} \in C_{s}\left(\mathbb{R}_{+}, X\right)$ and $a, \delta>0$ such that $e(\varepsilon)=c=\{\bar{\zeta}([0, t])\}_{t \in \mathbb{R}} \cup\{X\} \in \mathfrak{R}_{x}, \bar{\zeta}([0,1-\delta]) \subset \bar{B} \cup V, \bar{\zeta}(t) \notin \bar{B}$ for $1-\delta \leq t \leq a$ and $d(\bar{\zeta}([0, a]), X)<\varepsilon$. Let $T=\bigcup_{k=1}^{R} U_{k}$ be an $\varepsilon$-tube of $\bar{\zeta}_{\mid[0, a]}$ with $U_{1}=B$ (this can be easily arranged by redefining $\zeta$ inside $\bar{B} \cup V$ ). Using the boundary shrinking property one can find $h \in G_{B}, y \in Z$ and $\delta_{0}>0$ such that $h(W) \subset B\left(y, \delta_{0}\right)$ and $h(x)=x$. The $\delta_{0}$ can be chosen small enough so that Lemma 4.1 implies there is $g_{1} \in G_{T}$ with $g_{1}(x)=x$ and $g_{1}\left(B\left(y, \delta_{0}\right)\right)$ is inside a sufficiently small neighborhood of $\zeta(a)$ (in the sense of Lemma 6.3). Let $g=g_{1} \circ h$.

We will now show that $d(g(F), c)<2 \varepsilon$. It is enough to prove that for all $f \in F$ one has $d(g(f), c)<2 \varepsilon$. Let $f \in F$. If $f \in H$ then $S(f) \cap W \neq \emptyset$. If $f \notin H$, then clearly the same conclusion holds. Notice that $h(f) \in M_{x}$, $\operatorname{dist}\left(h\left(f_{\bar{Z}}\right), y\right)<\delta_{0}$ and $h\left(f_{\bar{Z}}\right) \subset B$. Finally, $\bar{\zeta}(0) \in g\left(f_{\bar{Z}}\right) \subset T$ is sufficiently close to $\bar{\zeta}(a)$ and therefore parts (1) \& (3) of Lemma 6.3 imply the desired conclusion.

We now turn to the case $N=\infty$. We claim that in this case $F$ is $3 \varepsilon$ fiber-like. Let $\left\{c_{i}\right\}_{i=1}^{L} \subset M_{x}$ be an $\varepsilon$-net of $M_{x}$. Choose $f_{i} \in F, i=1, \ldots, L$, so that $S\left(f_{i}\right) \cap S\left(f_{j}\right)=\emptyset$ for $i \neq j$. The idea now will be to approximate the $c_{i}$ s by $\overline{\mathbb{R}}_{+}$-chains $s_{i}$ and then act on the $f_{i}$ s with an element $g \in G$ such that $g\left(f_{i}\right)$ will approximate $s_{i}$. We will make an essential use of the fact that the $f_{i}$ s intersect $\partial Z$ in disjoint locations in order to construct the above-mentioned $g \in G$.

Choose $y_{i} \in S\left(f_{i}\right)$ and $\mu>0$ so that $\mu<\min \left\{d\left(S\left(f_{i}\right),\left[f_{j}\right]_{\bar{Z}}\right) \mid 1 \leq i<\right.$ $j \leq L\}$. As $X$ is locally connected one can choose open connected subsets $C_{i}$ with $y_{i} \in C_{i} \subset B\left(y_{i}, \mu\right) \cap(A \cup \bar{Z}), i=1, \ldots, L$, where we use the fact that $A \cup \bar{Z}$ is open. As $C_{i}$ are open and connected, one can find simple paths $\gamma_{i} \in C_{s}\left([0,1], C_{i}\right), i=1, \ldots, L$, such that $\gamma_{i}(0)=y_{i}$ and $\gamma_{i}(1) \in A$. Now invoke Lemma 5.4 (with $k=1, F_{1}=\cdots=F_{L}=\bar{Z}$ and $U_{1}=\cdots=U_{L}=$ $\bar{Z} \cup A)$ to find $a>0$ and $\left(s_{1}, \ldots, s_{L}\right) \in \mathfrak{R}_{*}^{L}$ with $r\left(s_{i}\right)=\left\{y_{i}\right\}, i=1, \ldots, L$, represented as $s_{i}=\left\{\xi_{i}([0, t])\right\}_{t \in \mathbb{R}_{+}} \cup\{X\}, i=1, \ldots, L$, so that there exists $\delta>0$ with $\xi_{i}([1-\delta, a]) \cap \bar{Z}=\emptyset,\left[\xi_{i}\right]_{\mid[0,1-\delta]}=\left[\gamma_{i}\right]_{\mid[0,1-\delta]}, d\left(X, \xi_{i}([0, a])\right)<\varepsilon / 2$ for $i=1, \ldots, L$ and

$$
d\left(c_{i}, s_{i}\right)<\varepsilon, \quad i=1, \ldots, L .
$$


While defining the $\xi_{i}$ one can construct disjoint $\varepsilon$-tubes $T_{i}=\bigcup_{k=1}^{q_{i}} U_{i}^{k}, i=$ $1, \ldots, L$, with $U_{i}^{1}=Z, U_{i}^{2}=C_{i}$ and $\zeta_{i}([0, a]) \subset T_{i}$. Notice that $Q_{i}=$ $\bigcup_{k=2}^{q_{i}} U_{i}^{k}$ is an $\varepsilon$-tube (for $\zeta_{\mid[1-\delta, a]}$ ) so one can choose $g_{i} \in G_{Q_{i}}$ with $g\left(y_{i}\right)=$ $\xi_{i}(a)$. Let $f_{i}^{\prime}$ be the initial segment of $f_{i}$ ending at $\left[f_{i}\right]_{\bar{Z}}$. By Lemma 6.3,

$$
d\left(g_{i}\left(f_{i}^{\prime}\right), s_{i}\right)<2 \varepsilon, \quad i=1, \ldots, L .
$$

Define $g=g_{1} \circ \cdots \circ g_{L}$. As $g_{i} \in G_{Q_{i}}$ we have $\left[g_{i}\right]_{\mid\left[f_{j}\right]_{\bar{Z}}}=$ Id for $1 \leq i<$ $j \leq L$. From this and (8.3) and (8.4) we conclude that $d\left(c_{i}, g\left(f_{i}\right)\right)<3 \varepsilon$ for $i=1, \ldots, L$. In particular $d\left(g(F), M_{x}\right)<3 \varepsilon$. Finally, if $H=\emptyset$ we choose $\{*\}=W \subsetneq \partial Z$ and repeat the same construction used in the case $H \neq \emptyset$ and $N=1$.

COROLlary 8.5. Under the assumptions of Lemma 8.4, if $F \in V\left(M_{x}\right)$ and $|F|<\infty$, then $F$ is point-like.

TheOrem 8.6. Let the Peano continuum $X$ be $S R I$ and let $(X, G)$ have the boundary shrinking property. Then the only minimal subspaces of $V(M)$ are:

(1) $\{M\}$,

(2) $\left\{M_{x}\right\}_{x \in X}$

(3) $\{\{c\} \mid c \in M\}$.

Proof. The $G$-invariance of all three subspaces is clear. The fact that $\{M\}$ and $\{\{c\} \mid c \in M\}$ are closed is trivial. The fact that $\left\{M_{x}\right\}_{x \in X}$ is closed is proven in Lemma 2.1. The minimality of $\{M\}$ is trivial. The minimality of $\left\{M_{x}\right\}_{x \in X}$ is a consequence of Lemma 2.1 and the transitivity of the action of $G$ on $X$ (Lemma 4.1). The minimality of $\{\{c\} \mid c \in M\}$ is a consequence of Theorem 6.5. To show that the given subspaces are the only minimal subspaces it is enough to show that any $F \in V(M)$ is either space-like, fiber-like or point-like. Let $F \in V(M)$. If $|r(F)|=\infty$, then by Lemma 8.1, $F$ is space-like. If $|r(F)| \in \mathbb{N}$ one can assume that $|r(F)|=1$. By Lemma $8.4, F$ is either point-like or fiber-like.

9. The strong proximality of $M$. The goal of this section is to prove that $M$ is proximal under the assumption of the previous section and strongly proximal under additional assumptions. Let us start with the definition of these two terms. $M$ is said to be proximal under $G$ if for any $c, f \in M$ one can find $g_{n} \in G$ such that $\lim _{n \rightarrow \infty} d\left(g_{n}(c), g_{n}(f)\right)=0$; and $M$ is strongly proximal under $G$ if for any Borel probability measure $\mu$ on $M$, there exists a sequence $\left(g_{n}\right) \subset G$ such that $\left[g_{n}\right]_{*}(\mu)$ converges to a measure concentrated at a singleton.

Theorem 9.1. Let the Peano continuum $X$ be SRI and let $(X, G)$ have the boundary shrinking property. Then $(M, G)$ is proximal. 
Proof. This can be proven using only the assumptions of Section 6, but here we will use instead the method of Lemma 8.4. Let $c, f \in M$. One can assume that $c, f \in M_{x}$ for some $x \in X$. Define $F=\{c, f\} \in V(M)$. As $|F|=2$, by Corollary $8.5, F$ is point-like, i.e., there exist $g_{n} \in G$ so that diam $g_{n}(F) \rightarrow 0$ as $n \rightarrow \infty$, which is equivalent to the proximality of the pair $(c, f)$.

Theorem 9.2. Let the Peano continuum $X$ be SRI and let $(X, G)$ have the boundary shrinking property. If $(X, G)$ is strongly proximal then $(M, G)$ is strongly proximal.

Proof. Let $\mu$ be a Borel probability measure on $M$. Let $r_{*}(\mu)$ be the projection of $\mu$ under the map $r: M \rightarrow X$. Using the strong proximality of $(X, G)$ one can assume that $r_{*}(\mu)(\{x\})=1$ for some $x \in X$. Let $\varepsilon>0$. We will prove that one can find $g \in G$ and $c \in M$ such that $g_{*}(\mu)(B(c, 2 \varepsilon))>$ $1-\varepsilon$. By standard compactness arguments this will show $(M, G)$ is strongly proximal.

Using property (BSP) of $(X, G)$ choose $Z$ with the $(G, \varepsilon / 2, x)$-boundary shrinking property such that $x \in Z \subset B(x, \varepsilon / 2)$. Let $f \in M_{x}$. Define $S(f)=$ $f_{\bar{Z}} \cap \partial Z$. Let $W \subsetneq \partial Z$ be a closed subset. Repeating an argument appearing in Lemma 8.4, we can find $c \in \mathfrak{R}_{x}$ and $h_{W} \in G$ such that $f \in M_{x}$ with $S(f) \cap$ $W \neq \emptyset$ implies $d\left(h_{W}(f), c\right)<2 \varepsilon$. Define $E_{W}=\left\{f \in M_{x} \mid S(f) \cap W \neq \emptyset\right\}$ and $F_{W}=\left\{f \in M_{x} \mid d\left(h_{W}(f), c\right)<2 \varepsilon\right\}$. Notice that $F_{W}$ is open in $M_{x}$ and $E_{W} \subset F_{W}$. Another useful property is that if $W_{0}, W_{1} \subsetneq \partial Z$ are closed subsets such that $\partial Z \backslash W_{0}$ and $\partial Z \backslash W_{1}$ are disjoint then $M_{x} \backslash F_{W_{0}}$ and $M_{x} \backslash F_{W_{1}}$ are also disjoint. Indeed, if $f \in M_{x} \backslash F_{W_{i}}$ then $S(f) \subset \partial Z \backslash W_{i}$. This implies $S(f) \cap W_{\bar{i}} \neq \emptyset$, i.e. $f \in E_{W_{\bar{i}}} \subset F_{W_{\bar{i}}}$, which implies $f \notin M_{x} \backslash F_{W_{\bar{i}}}$ (here we use the convention $\overline{0}=1, \overline{1}=0$ ). Let $n \in \mathbb{N}$ with $1 / n \leq \varepsilon$. As $\partial Z$ is connected and has at least two points one can choose $n$ non-empty pairwise disjoint open subsets $O_{1}, \ldots, O_{n} \subset \partial Z$. Define $W_{i}=\partial Z \backslash O_{i}, i=1, \ldots, n$. Then the closed sets $M_{x} \backslash F_{W_{i}}, i=1, \ldots, n$, are pairwise disjoint. Hence there exist $1 \leq j \leq n$ so that $\mu\left(M_{x} \backslash F_{W_{j}}\right) \leq 1 / n<\varepsilon$. Thus $\mu\left(F_{W_{j}}\right)>1-\varepsilon$, i.e. $\left[h_{W_{j}}\right]_{*}(\mu)(B(c, 2 \varepsilon))>1-\varepsilon$.

We call $(X, G)$ base-wise shrinkable if $X$ has a basis $\left\{U_{\alpha}\right\}_{\alpha \in A}$ (called a shrinkable basis) such that for any pair of open subsets $V \subset \bar{V} \subset U_{\alpha}, W \subset U_{\alpha}$ there is $g \in G_{U_{\alpha}}$ with $g(V) \subset W$. It turns out that for such spaces one can prove strong proximality.

Lemma 9.3. Suppose $X$ is a Peano continuum such that for each connected open set $U \subset X$ and each $p \in X$ the set $U \backslash\{p\}$ is connected. If $(X, G)$ is base-wise shrinkable then $(X, G)$ is strongly proximal.

Proof. Let $\mathfrak{M}(X)$ be the space of Borel probability measures of $X$. Let $\varepsilon>0$. We will show there exists an open set $U_{\varepsilon}$ with $\operatorname{diam} U_{\varepsilon}<\varepsilon$ and $g_{\varepsilon} \in G$ 
such that $\mu\left(g_{\varepsilon}\left(U_{\varepsilon}\right)\right)>1-\varepsilon$. Cover $X$ by elements from a shrinkable basis $\left\{U_{k}\right\}_{k=1}^{N-1}$ so that diam $U_{k}<\varepsilon$ for $k=1, \ldots, N-1$. Assume without loss of generality that there exists a non-empty open subset $U_{N} \subset U_{1} \backslash \bigcup_{k=2}^{N-1} U_{k}$. Define $U_{k}^{r}=\left\{y \in U_{k} \mid \operatorname{dist}\left(y, U_{k}^{c}\right)>1 / r\right\}, r \in \mathbb{N}, 1 \leq k \leq N$. Notice that $U_{k}=\bigcup_{r=1}^{\infty} U_{k}^{r}$. If there is $g \in G$ such that $\mu\left(g\left(U_{1}\right)\right)>1-\varepsilon$, we are done. Assume otherwise. Let $s=\sup _{g \in G} \mu\left(g\left(U_{N}\right)\right)$. As $U_{N} \subset U_{1}$, we have $s \leq 1-\varepsilon$. Using the fact that $U_{1}$ is part of a shrinkable basis one can assume that $s-(1-s) / 2 N<\mu\left(U_{N}\right) \leq s$. As $\mu\left(U_{1}\right) \leq 1-\varepsilon$, there is $2 \leq k \leq N-1$ such that $\mu\left(U_{k}\right)>(1-s) / 2 N$, in particular there is $l \in \mathbb{N}$ with $\mu\left(U_{k}^{l}\right)>(1-s) / 2 N$. Choose $q \in \mathbb{N}$ so that $s-(1-s) / 2 N<\mu\left(U_{N}^{q}\right) \leq s$. As $X$ is arcwise connected there is $p \in C_{s}\left([0,1], X \backslash U_{N}^{q}\right)$ such that $p(0) \in U_{k}$ and $p(1) \in U_{N}$. Let $T$ be a $\delta$-tube for $p$ for some $\delta>0$ so that $T \subset X \backslash U_{N}^{q}$. Using Lemma 4.1 find $h \in G_{T}$ such that $h(p(0))=p(1)$. Find an open subset $p(0) \in Z \subset U_{k}$ such that $h(Z) \subset U_{N}$. As $(X, G)$ is base-wise shrinkable one can find $e \in G_{U_{k}}$ (in particular $e_{\mid U_{N}}=\mathrm{Id}$ ) with $e\left(U_{k}^{l}\right) \subset Z$. Define $g=$ $(h \circ e)^{-1}$. It is easy to see that $\mu\left(g\left(U_{N}\right)\right)>s-(1-s) / 2 N+(1-s) / 2 N=s$, contrary to the definition of $s$. We conclude that $\sup _{g \in G} \mu\left(g\left(U_{1}\right)\right)>1-\varepsilon$.

From Theorems 9.2 and 9.3 we have:

Theorem 9.4. Let the Peano continuum $X$ be SRI and let $(X, G)$ have the boundary shrinking property. If $(X, G)$ is base-wise shrinkable then $(M, G)$ is strongly proximal.

10. On the structure of $V\left(M\left(S^{2}\right)\right)$. Let $X=S^{2}$, the two-dimensional sphere. It is strongly arcwise-inseparable but not strongly $\mathbb{R}$-inseparable. One may ask if Theorem 8.6 still holds in this setting. The following theorem answers this question negatively.

Theorem 10.1. Let $X=S^{2}$. There exists $F \subseteq V(M)$ which is neither point-like, nor space-like, nor fiber-like.

Proof. To simplify notation assume $X=S^{2}=\left\{(x, y, z) \mid x^{2}+y^{2}+z^{2}=1\right\}$ $\subset \mathbb{R}^{3}$. Let $p=(0,0,-1)$ and $n=(0,0,1)$ be the "south" and "north" poles of $X$. Let $T_{p}$ be the tangent space of $X$ at $p$. Let $\widehat{x} \in T_{p}$ be the unit tangent vector in the direction of the $x$-axis. We are going to define a family of curves $q_{(x, \theta) \rightarrow n}$, where $x \in X$ and $\theta=\theta(x) \in[0,2 \pi]$ represents an angle.

We start by defining for $\theta \in[0,2 \pi]$ the curve $q_{(p, \theta) \rightarrow n}:[0,1] \rightarrow X$ as the unique geodesic of $X$ with $q_{(p, \theta) \rightarrow n}(0)=p, q_{(p, \theta) \rightarrow n}(1)=n$ and $\angle\left(\widehat{x}, \dot{q}_{(p, \theta) \rightarrow n}(0)\right)=\theta$. Fix $t \in(0,1]$ and $\theta \in[0,2 \pi]$. Let $x=q_{(p, \theta) \rightarrow n}(t)$ and denote by $q_{(x, \theta) \rightarrow n}:[0,1] \rightarrow X$ the unique geodesic of $X$ with $q_{(x, \theta) \rightarrow n}(0)=$ $x, q_{(x, \theta) \rightarrow n}(1)=n$ and $q_{(x, \theta) \rightarrow n}([0,1]) \subset q_{(p, \theta) \rightarrow n}([0,1])$. Given $c=\left\{c_{\alpha}\right\}_{\alpha \in A}$ $\in M$ and $l \in C([0, h], X)$ with $\{l(h)\}=r(c)$ define the "concatenated maxi- 
mal chain":

$$
s(l, c)=\left\{l([0, t]\}_{t \in[0, h]} \cup\left\{l([0, h]) \cup c_{\alpha}\right\}_{\alpha \in A} .\right.
$$

Notice that $s(l, c) \in M_{l(0)}$. Let $c=\left\{\bar{B}(n, \varepsilon\}_{\varepsilon \geq 0} \in M\right.$. Define

$$
F=\left\{s\left(q_{\left(q_{(p, \theta) \rightarrow n}(t), \theta\right) \rightarrow n}, c\right)\right\}_{(t, \theta) \in[0,1] \times[0,2 \pi]} .
$$

It is easy to see that $F \in V(M)$. Indeed, given a converging sequence $\left\{s\left(q_{\left(x_{i}, \theta_{i}\right) \rightarrow n}, c\right)\right\}_{i=1}^{\infty} \subseteq F$ there are $x^{*} \in X$ and $\theta^{*} \in[0,2 \pi)$ so that by passing to a subsequence $x_{i} \rightarrow x^{*}$ and $\theta_{i} \rightarrow \theta^{*} \bmod 2 \pi$ as $i \rightarrow \infty$. Clearly $s\left(q_{\left(x_{i}, \theta_{i}\right) \rightarrow n}, c\right) \rightarrow s\left(q_{\left(x_{*}, \theta_{*}\right) \rightarrow n}, c\right)$ as $i \rightarrow \infty$ for the original sequence. Notice that $r(F)=X$, so $F$ is neither point-like nor fiber-like.

We will now show $F$ is not space-like. Let $e:[0,2 \pi] \rightarrow X$ be the "equatorial" great circle $e(t)=(\cos (\pi+t), \sin (\pi+t), 0)$. Let $w:[0,2 \pi] \rightarrow$ $X$ be the "Greenwich" great circle $w(t)=(0, \sin (-t), \cos (-t))$. Let $m_{1} \in$ $M_{(-1,0,0)}, m_{2} \in M_{(0,0,1)}$ be arbitrary elements. Define $c_{1}=s\left(e, m_{1}\right) \in M_{e(0)}$ and $c_{2}=s\left(w, m_{2}\right) \in M_{w(0)}$.

We will show that for any $A \in \mathrm{Cl}_{V(M)}(G F)$ one has $\left\{c_{1}, c_{2}\right\} \notin A$. In particular $M \notin \mathrm{Cl}_{V(M)}(G F)$. Our proof is based on the following observation: if $E, D \in C(X)$, then by the Jordan separation theorem there exists $\varepsilon_{0}>0$ such that $D \subset B\left(w([0, \pi]), \varepsilon_{0}\right)$ and $E \subset B\left(e([0, \pi]), \varepsilon_{0}\right)$ imply that $E \cap D \neq \emptyset$ and in fact $E \cap D \subset I$ where $I=B\left(w([0, \pi]), \varepsilon_{0}\right) \cap B\left(e([0, \pi]), \varepsilon_{0}\right)$. We choose $0<\varepsilon<\min \left\{\varepsilon_{0}, \frac{1}{2} d(e(0), w(0))\right\}$.

Assume for contradiction that there exist $g \in G$ and $f_{1}, f_{2} \in F$ such that $d\left(g\left(f_{i}\right), c_{i}\right)<\varepsilon$ for $i=1,2$. In particular there exist $Y_{i} \in f_{i}$ for $i=1,2$ such that $d\left(g\left(Y_{1}\right), e([0, \pi])\right)<\varepsilon$ and $d\left(g\left(Y_{2}\right), w([0, \pi])\right)<\varepsilon$. We also have $d\left(r\left(g\left(Y_{1}\right)\right), e(0)\right)<\varepsilon$ and $d\left(r\left(g\left(Y_{2}\right)\right), w(0)\right)<\varepsilon$, which implies that $r\left(g\left(Y_{1}\right)\right) \neq r\left(g\left(Y_{2}\right)\right)$. As $g\left(Y_{1}\right)$ and $g\left(Y_{2}\right)$ intersect, i.e. $\emptyset \neq g\left(Y_{1}\right) \cap$ $g\left(Y_{2}\right) \in I$, we conclude that $Y_{1}=q_{\left(x_{1}, \theta_{1}\right) \rightarrow n}([0,1)) \cup B\left(n, \varepsilon_{1}\right)$ and $Y_{1}=$ $q_{\left(x_{2}, \theta_{2}\right) \rightarrow n}([0,1)) \cup B\left(n, \varepsilon_{2}\right)$ for $x_{1} \neq x_{2}$ and without loss of generality $\varepsilon_{1} \geq$ $\varepsilon_{2} \geq 0$. Notice that $B\left(e([0, \pi]), \varepsilon_{0}\right) \backslash I$ has two components. Let $J_{1}$ be the component with $r\left(g\left(Y_{1}\right)\right) \subset J_{1}$. Similarly, let $J_{2}$ be the component of $B\left(w([0, \pi]), \varepsilon_{0}\right) \backslash I$ with $r\left(g\left(Y_{2}\right)\right) \subset J_{2}$. We conclude that

$$
\begin{aligned}
& g\left(f_{1}\right)=s\left(\left\{g \circ q_{\left(x_{1}, \theta_{1}\right) \rightarrow n}([0, t])\right\}_{t \in[0,1]}, g(c)\right), \\
& g\left(f_{2}\right)=s\left(\left\{g \circ q_{\left(x_{2}, \theta_{2}\right) \rightarrow n}([0, t])\right\}_{t \in[0,1]}, g(c)\right),
\end{aligned}
$$

where $q_{\left(x_{i}, \theta_{i}\right) \rightarrow n}([0,1]) \subset J_{i} \cup I, i=1,2$. In other words, until $g\left(f_{1}\right)$ and $g\left(f_{2}\right)$ "meet" they are confined to $J_{1} \cup I$ and $J_{2} \cup I$ respectively. After they "meet" they develop identically (which corresponds to the $g(c)$ part of the concatenation). This is a clear contradiction to $d\left(g\left(f_{i}\right), c_{i}\right)<\varepsilon$ for $i=1,2$ for $\varepsilon$ small enough.

11. Manifolds and the Hilbert cube. In this section we present classes of examples to which one can apply the results of the article. 
Theorem 11.1. Let $X$ be a two-dimensional closed topological manifold and $G$ a locally transitive group acting on $X$. Then $(M(X), G)$ is the unique minimal subspace of $(\Phi(X), G)$. Moreover, the universal minimal space $\left(U_{G}, G\right)$ is not transitive.

Proof. As $X$ is a closed topological manifold, $X$ is a Peano continuum. By assumption $G$ acts transitively on $X$. By Lemma A.1, $X$ is strongly arcwiseinseparable. These facts enable us to conclude by Theorem 6.5 and Corollary 6.6 that $(M(X), G)$ is minimal and $\left(U_{G}, G\right)$ is not transitive. Moreover, as $X$ is a closed topological manifold, it is easy to see $X$ has the telescoping annuli property. This implies by Theorem 7.2 that $(M(X), G)$ is the only minimal subspace of $(\Phi(X), G)$.

THEOREM 11.2. Let $X$ be a closed topological manifold of dimension $n \geq 3$ and $G$ a subgroup of the homeomorphism group of $X$. If $G \supset \operatorname{Homeo}_{0}(X)$ or $X$ has a smooth structure such that $G \supset \operatorname{Diffeo}_{0}(X)$, then $(M(X), G)$ is minimal and strongly proximal and the unique minimal subspace of $(\Phi(X), G)$. The only minimal subspaces of $(V(M(X)), G)$ are $\{M(X)\},\left\{M(X)_{x}\right\}_{x \in X}$ and $\{\{c\} \mid c \in M(X)\}$. The universal minimal space $\left(U_{G}, G\right)$ is not transitive.

Proof. Notice that by the discussion of locally transitive group actions at the end of Section 3, $G \supset \operatorname{Homeo}_{0}(X)$ or $G \supset \operatorname{Diffeo}_{0}(X)$ imply that $G$ acts locally transitively on $X$. Using Theorem 11.1 we conclude $\left(U_{G}, G\right)$ is not transitive and $(M(X), G)$ is the unique minimal subspace of $(\Phi(X), G)$. By Lemma A.3, $X$ is strongly $\mathbb{R}$-inseparable. By Lemma A.5, $(X, G)$ has the boundary shrinking property. The last two facts enable us to conclude that the only minimal subspaces of $(V(M(X)), G)$ are $\{M(X)\},\left\{M(X)_{x}\right\}_{x \in X}$ and $\{\{c\} \mid c \in M(X)\}$. Finally, it is easy to verify that $(X, G)$ is base-wise shrinkable, which implies $(M(X), G)$ is strongly proximal

Recall that the Hilbert cube is defined to be $Q=[-1,1]^{\mathbb{N}}$, equipped with the metric $d\left(\left(x_{n}\right)_{n=1}^{\infty},\left(y_{n}\right)_{n=1}^{\infty}\right)=\max \left\{\left|x_{n}-y_{n}\right| / n \mid n=1,2, \ldots\right\}$.

Theorem 11.3. Let $G=\operatorname{Homeo}(Q)$. The $G$-space $(M(Q), G)$ is minimal and strongly proximal and the unique minimal subspace of $(\Phi(Q), G)$. The only minimal subspaces of $(V(M(Q)), G)$ are $\{M(Q)\},\left\{M(Q)_{x}\right\}_{x \in X}$ and $\{\{c\} \mid c \in M(Q)\}$. The universal minimal space $\left(U_{G}, G\right)$ is not 1-transitive.

Proof. The Hilbert cube $Q$ is metric, compact, connected and locally connected. $G$ acts locally transitively by Lemma A.2. By Lemma A.4, $Q$ has the telescoping annuli property. By Lemma A.3, $Q$ is strongly $\mathbb{R}$-inseparable. Using Theorem 6.5, Theorem 7.2 and Corollary 6.6 we conclude that $(M(Q), G)$ is the unique minimal subspace of $(\Phi(Q), G)$ and $\left(U_{G}, G\right)$ is not transitive.

We now proceed to prove that the only minimal subspaces of $(V(M(Q)), G)$ are $\{M(Q)\},\left\{M(Q)_{x}\right\}_{x \in X}$ and $\{\{c\} \mid c \in M(Q)\}$. The natural approach would be to use Theorem 8.6. However, in order to use it one has to show 
that $(Q, G)$ has the boundary shrinking property. Unfortunately, we have not been able to do that (see Question 12.2). A careful reading shows that the boundary shrinking property is used in the proof of Theorem 8.6 only via the use of Lemma 8.4. We give in Lemma A.7 a separate proof for $(Q, G)$ and thus achieve the above mentioned result. By Lemma A.8, $(M(Q), G)$ is strongly proximal.

12. Open questions. We are ultimately interested in finding all minimal subspaces of $V^{n}(X)$ for $n \in \mathbb{N}$; unfortunately, this turned out to be too difficult and we leave it as a question to the reader:

QUESTION 12.1. Can one characterize all minimal subspaces of $V^{n}(X)$, $n \geq 2$ ?

A natural way to prove the results of Section 8 for $(Q$, Homeo $(Q))$ is to show that $(Q, \operatorname{Homeo}(Q))$ has the $(\mathrm{BSP})$ property. Unfortunately, we are unable to settle the following question:

Question 12.2. Does $(Q, \operatorname{Homeo}(Q))$ have the boundary shrinking property?

In the Introduction we mentioned Open Questions $28 \& 29$ of [Pes05] which ask for an explicit description of the universal minimal space of the group of homeomorphisms Homeo $(X), X$ being a closed manifold of dimension 2 or higher or the Hilbert cube. In view of our results we reformulate these questions as follows:

QUESTION 12.3. Is the universal minimal space for the group $\operatorname{Homeo}(X)$, $X$ being a closed manifold of dimension 3 or higher or the Hilbert cube, equal to the space $M(X)$ ?

Appendix. The appendix contains various topological results used in Sections 3 and 11. The first three are reformulations of facts which are well known, sometimes in greater generality. For the reader's convenience we provide, however, detailed arguments or bibliographical hints.

Lemma A.1. If $X$ is a two-dimensional closed topological manifold then $X$ is strongly arcwise-inseparable.

Proof. Let $U \subset X$ be open and connected and $J \subset U$ be an arc. The connectedness of $U \backslash J$ is known in greater generality when $J$ is a cell-like compact subset of $X$, that is, one which can be contracted to a point in each of its neighborhoods. See Corollary 4B on p. 121 of [Dav86].

Lemma A.2. Homeo $(Q)$ is locally transitive and $n$-transitive for all $n \in \mathbb{N}$.

Proof. In [BP75, p. 145] in the proof of Proposition 8.1 Bessaga and Pełczyński show that $\operatorname{Homeo}(Q)$ is strongly locally homogeneous, i.e. for each 
$x \in Q$ there exists a basis $\left\{V_{i}\right\}$ of open neighborhoods such that $\operatorname{Homeo}(Q)_{V_{i}}$ acts transitively on $V_{i}$ for each $i$. Thus $\operatorname{Homeo}(Q)$ is locally transitive and, by Theorem 4.1 , also $n$-transitive.

Lemma A.3. If $X$ is a closed topological manifold of dimension $n \geq 3$ or the Hilbert cube, then $X$ is strongly $\mathbb{R}$-inseparable.

Proof. Let $J$ be an arc in $X$ and $U \subset X$ be a connected open set. To establish that $U \backslash J$ is connected we consider three cases:

1) $X$ is an $n$-manifold and $U$ is homeomorphic to $\mathbb{R}^{n}$, where $n \geq 3$. Then $U \backslash S$ is connected for any closed set $S \subset X$ of dimension not greater than $n-2$; see Theorem 1.8.13 in [Eng78]. In particular, this applies to $S=J$.

2) $X=Q$. By the definition of the product topology of $Q$ there exist $n \geq 3$ and a chart $V \subset \prod_{k=1}^{n}(-1,1)_{k}, V \cong \mathbb{R}^{n}$, such that $V \times \prod_{k>n}[-1,1]_{k} \subset U$. Given $x, y \in U$ there exists by the lemma above an $f \in \operatorname{Homeo}(Q)_{U}$ such that $f(x)$ and $f(y)$ belong to $V \times\{0\}$. By 1) above, the set $V \times\{0\} \backslash f(J)$ is connected and hence there is an arc $K$ in it connecting $f(x)$ to $f(y)$. Clearly, $f^{-1}(K)$ is an arc in $U \backslash J$ connecting $x$ to $y$. Since $x, y$ are arbitrary points of $U \backslash J$, this set is connected.

3) The case where $X$ is an $n$-manifold but $U$ is not homeomorphic to $\mathbb{R}^{n}$ follows from 1$)$ in precisely the same manner, using the 2-transitivity of $\operatorname{Homeo}(X)_{U}$.

Lemma A.4. $Q$ has the telescoping annuli property.

Proof. To show that there is a $(1, \varepsilon)$ annuli telescope around a given point $x \in Q$ we first note that by Lemma A.2 we can assume that $x=$ $(0,0, \ldots)$. As in the proof above, there exists a set of diameter smaller than $\varepsilon$ which contains 0 and is of the form $V \times \prod_{k>n}[-1,1]_{k}$ for some open $V \subset$ $\prod_{k=1}^{n}(-1,1)$. Let $\{B, U\}$ be a $(1, \delta)$ annuli telescope in $V$ around $(0, \ldots, 0)$ for some $\delta>0$ and the Euclidean metric of $V$. Define $B^{\prime}=B \times \prod_{k>n}[-1,1]_{k}$ and $U^{\prime}=U \times \prod_{k>n}[-1,1]_{k}$. It is clear one can choose $\delta$ so small that $\left\{B^{\prime}, U^{\prime}\right\}$ is an $(1, \varepsilon)$ annuli telescope around $x=0$.

Lemma A.5. Let $X$ be a closed topological manifold of dimension $n \geq 2$ and $G$ a subgroup of $\operatorname{Homeo}(X)$. If $G \supseteq \operatorname{Homeo}_{0}(X)$ or $X$ is a smooth manifold and $G \supseteq \operatorname{Diffeo}_{0}(X)$ then $(X, G)$ has the boundary shrinking property.

Proof. Let $x \in X$ and $\varepsilon>0$. Since $X$ is a manifold one can find a chart $C \cong \mathbb{R}^{n}$ so that $x \in C \subset B(x, \varepsilon / 2)$. Let $A$ and $E$ be open balls (in the Euclidean metric of $C$ ) with center $x$ such that $\bar{A} \subset E \subset C$. It is easy to see that $A$ has the $(G, \varepsilon, x)$-boundary shrinking property, with $E \backslash A$ being a set $\varepsilon$-encircling $\bar{A}$.

Lemma A.6. For $n \geq 3$, let $x \in \operatorname{Int}\left(I^{n}\right)$ and $F \in V\left(M_{x}\right)$. If $F$ is not point-like then it is fiber-like. 
Proof. This is a simple generalization of the techniques used in this article. One uses strongly the fact that $x \in \operatorname{Int}\left(I^{n}\right)$. The idea is that all constructions in the first nine sections can be done inside $\operatorname{Int}\left(I^{n}\right)$ and therefore the statement follows from the same statement for $S^{n}$ (proven in Lemma 8.4 applied to $X=S^{n}$ ).

For $n \in \mathbb{N}$ it will be convenient to denote the product $\prod_{i=1}^{n}[-1,1]_{i}$ by $I^{n}$, $\prod_{i=n}^{\infty}[-1,1]_{i}$ by $I_{n}^{\infty}$ and the standard projection of $Q$ onto $I^{n}$ by $\pi_{n}$. Given a homeomorphism $g$ of $I^{n}$ we write $\widetilde{g}$ for the homeomorphism of $Q$ which composed with $\pi_{n}$ is equal to $g$ and composed with the projection onto $I_{n+1}^{\infty}$ equals this projection. In the following lemmas it is convenient to use the metrics

$$
d\left(\left(x_{m}\right)_{m=1}^{n},\left(y_{m}\right)_{m=1}^{n}\right)=\max \left\{\left|x_{m}-y_{m}\right| / m \mid m=1, \ldots, n\right\} \quad \text { on } I^{n}
$$

and

$$
d\left(\left(x_{m}\right)_{m=1}^{\infty},\left(y_{m}\right)_{m=1}^{\infty}\right)=\max \left\{\left|x_{m}-y_{m}\right| / m \mid m=1,2, \ldots\right\} \quad \text { on } Q .
$$

Lemma A.7. Let $x=(0,0, \ldots) \in Q$. Let $F \in V\left(Q_{x}\right)$. Then $F$ is either point-like or fiber-like.

Proof. According to Lemma A.6 for each $n \in \mathbb{N}, \pi_{n}(F) \in V\left(M_{\pi_{n}(x)}\left(I^{n}\right)\right)$ is either point-like or fiber-like. Then either (1) there exists a sequence $n_{1}<$ $n_{2}<\cdots$ of integers such that $\pi_{n_{i}}(F)$ is fiber-like, or (2) there exists a sequence $n_{1}<n_{2}<\cdots$ of integers such that $\pi_{n_{i}}(F)$ is point-like.

In case (1), we claim $F$ is fiber-like. Let $\varepsilon>0$, and let $\left\{c_{i}\right\}_{i=1}^{L} \subset M_{x}(Q)$ be an $\varepsilon$-net of $M_{x}(Q)$. Choose $i \in \mathbb{N}$ such that $1 / n_{i}<\varepsilon$. Find $g \in \operatorname{Homeo}\left(I^{n_{i}}\right)$ with $d\left(g\left(\pi_{n_{i}}(F)\right), M_{\pi_{n_{i}}(x)}\left(I^{n_{i}}\right)\right)<\varepsilon$. In particular there exists $\left\{f_{k}\right\}_{k=1}^{L} \subset F$ such that $d\left(g\left(\pi_{n_{i}}\left(f_{k}\right)\right), \pi_{n_{i}}\left(c_{k}\right)\right)<\varepsilon$ for $k=1, \ldots, L$. As $1 / n_{i}<\varepsilon$, one concludes that $d\left(\tilde{g}\left(f_{k}\right), c_{k}\right)<\varepsilon$. This implies $d\left(\tilde{g}(F), M_{x}(Q)\right)<2 \varepsilon$.

In case (2), we claim $F$ is point-like. As in the previous case, fix $i \in \mathbb{N}$ such that $1 / n_{i}<\varepsilon$. Find $g \in \operatorname{Homeo}\left(I^{n_{i}}\right)$ and $c=\left\{c_{\alpha}\right\}_{\alpha \in A} \in M_{\pi_{n_{i}}(x)}\left(I^{n_{i}}\right)$ such that $d\left(g\left(\pi_{n_{i}}(F)\right),\{c\}\right)<\varepsilon$. Let $p \in M_{x}(Q)$ with $\left\{c_{\alpha} \times I_{n_{i}+1}^{\infty}\right\}_{\alpha \in A} \subset p$ (this corresponds to finding $\left\{e_{\beta}\right\}_{\beta \in B} \in M_{x}\left(\pi_{n_{i}}(x) \times I_{n_{i}+1}^{\infty}\right)$ and defining $P=$ $\left.\left\{e_{\beta}\right\}_{\beta \in B} \cup\left\{c_{\alpha} \times I_{n_{i}+1}^{\infty}\right\}_{\alpha \in A}\right)$. As $1 / n_{i}<\varepsilon$, one concludes that $d(\widetilde{g}(F),\{p\})<\varepsilon$. Indeed, fix $f \in F$. Let $R \in f \in F$. Find $C \in c$ such that $d\left(\pi_{n_{i}}(R), C\right)<\varepsilon$, which implies $d\left(R, C \times I_{n_{i}+1}^{\infty}\right)<\varepsilon$. Let $P \in p$. If $P=C \times I_{n_{i}+1}^{\infty}$ for some $C \in c$ then one can find $R \in f$ with $d\left(R, C \times I_{n_{i}+1}^{\infty}\right)<\varepsilon$. If this is not the case we must have $P \subseteq r(c) \times I_{n_{i}+1}^{\infty}$. As $d\left(r(c), r\left(\pi_{n_{i}}(f)\right)\right)=d\left(r(c), \pi_{n_{i}}(r(f))\right)<\varepsilon$, we conclude that $d(P, r(f))<\varepsilon$.

To show that $(M(Q)$, Homeo $(Q))$ is strongly proximal, we first prove the following lemma:

Lemma A.8. $(Q, \operatorname{Homeo}(Q))$ is strongly proximal. 
Proof. Clearly it is enough to show that $\left(\operatorname{Homeo}\left(I^{n}\right), I^{n}\right)$ is strongly proximal for each $n \in \mathbb{N}$. Fix $n \in \mathbb{N}$. Notice that $\partial I^{n}$ and $\operatorname{Homeo}\left(I^{n}\right)$ have a property which is very similar to (albeit weaker than) the boundary shrinking property. Indeed, for any closed $W \subsetneq \partial I^{n}$ and any $y \in \partial I^{n}$ and $\delta>0$ there is $h \in \operatorname{Homeo}\left(I^{n}\right)$ such that $h(W) \subset B(y, \delta)$. Let us call this the weak boundary shrinking property. Let $\mu \in \mathfrak{M}\left(I^{n}\right), \varepsilon>0$ and $N \in \mathbb{N}$ with $1 / N<\varepsilon$. Set $b=\mu\left(\partial I^{n}\right)$. By choosing $N$ disjoint open subsets of $\partial I^{n}$, considering their complements and using the weak boundary shrinking property we find $h_{1} \in \operatorname{Homeo}\left(I^{n}\right)$ and $y \in \partial I^{n}$ such that $\mu\left(h_{1}(B(y, \varepsilon)) \cap \partial I^{n}\right)>(1-\varepsilon) b$. Let $P_{k}=[-1+1 / k, 1-1 / k]^{n}, k \in \mathbb{N}$. Notice that $I^{n}=\bigcup_{k=1}^{\infty} P_{k}$. As $h_{1}\left(\operatorname{Int}\left(I^{n}\right)\right)=\operatorname{Int}\left(I^{n}\right)$, there is $q \in \mathbb{N}$ such that $\mu\left(h_{1}\left(P_{q}\right)\right)>(1-\varepsilon)(1-b)$. Again relying on the structure of $\operatorname{Homeo}\left(I^{n}\right)$ we can find $h_{2} \in \operatorname{Homeo}\left(I^{n}\right)$ with $\left[h_{2}\right]_{\mid \partial I^{n}}=\mathrm{Id}$ and $h_{2}\left(h_{1}\left(P_{q}\right)\right) \subset B(y, \varepsilon)$. Let $h=h_{2} \circ h_{1}$. We conclude that

$$
\mu(h(B(y, \varepsilon)))>(1-\varepsilon) b+(1-\varepsilon)(1-b)=(1-\varepsilon) .
$$

We cannot use Theorem 9.2 directly as we have not shown that $(Q, \operatorname{Homeo}(Q))$ has the $(\mathrm{BSP})$ property. Instead we prove directly:

Theorem A.9. $(M(Q)$, Homeo $(Q))$ is strongly proximal.

Proof. Let $\mu \in \mathfrak{M}(M(Q))$. Let $r_{*}(\mu)$ be the projection of $\mu$ under the map $r: M \rightarrow Q$. Using the strong proximality of $(Q$, Homeo $(Q))$ one can assume that $r_{*}(\mu)(\{x\})=1$ for $x=(0,0, \ldots)$. Let $N \in \mathbb{N}$ with $1 / N<\varepsilon$. Using the ideas in the proof of Lemma A.6 and Theorem 9.2, one can find $g_{N} \in \operatorname{Homeo}\left(I^{N}\right)$ and $c_{N} \in M\left(I^{N}\right)$ such that $\left[g_{N} \circ \pi_{N}\right]_{*}(\mu)\left(B\left(c_{N}, 4 \varepsilon\right)\right)>$ $1-\varepsilon$. Using the ideas in the proof of Lemma A.7 one finds $c \in M(Q)$ such that $\widetilde{g}_{*}(\mu)(B(c, 5 \varepsilon))>1-\varepsilon$.

\section{References}

[BP75] C. Bessaga and A. Pełczyński, Selected Topics in Infinite-Dimensional Topology, Monografie Mat. 58, PWN-Polish Sci. Publ., Warszawa, 1975.

[Dav86] R. J. Daverman, Decompositions of Manifolds, Pure Appl. Math. 124, Academic Press, Orlando, FL, 1986.

[Eng78] R. Engelking, Dimension Theory, North-Holland Math. Library 19, NorthHolland, Amsterdam, 1978.

[GW02] E. Glasner and B. Weiss, Minimal actions of the group $\mathbb{S}(\mathbb{Z})$ of permutations of the integers, Geom. Funct. Anal. 12 (2002), 964-988.

[GW03] - - - The universal minimal system for the group of homeomorphisms of the Cantor set, Fund. Math. 176 (2003), 277-289.

[IN99] A. Illanes and S. B. Nadler, Jr. Hyperspaces, Monogr. Textbooks Pure Appl. Math. 216, Dekker, New York, 1999.

[KPT05] A. S. Kechris, V. G. Pestov, and S. Todorcevic, Frä̈ssé limits, Ramsey theory, and topological dynamics of automorphism groups, Geom. Funct. Anal. 15 (2005), 106-189. 
[Mun75] J. R. Munkres, Topology: a First Course, Prentice-Hall, Englewood Cliffs, NJ, 1975.

[Pes98] V. G. Pestov, On free actions, minimal flows, and a problem by Ellis, Trans. Amer. Math. Soc. 350 (1998), 4149-4165.

[Pes05] - Dynamics of Infinite-Dimensional Groups and Ramsey-Type Phenomena, Publ. Mat. IMPA, IMPA, Rio de Janeiro, 2005.

[Usp00] V. Uspenskij, On universal minimal compact G-spaces, Topology Proc. 25 (2000), 301-308.

Institute of Mathematics

The Hebrew University

Jerusalem, Israel

E-mail: gyonatan@math.huji.ac.il

Received 25 September 2005;

in revised form 20 November 2006 and 28 January 2008 